\title{
SINGULAR SOLUTIONS TO A SEMILINEAR BIHARMONIC EQUATION WITH A GENERAL CRITICAL NONLINEARITY
}

\author{
RUPERT L. FRANK AND TOBIAS KÖNIG
}

To V. Maz'ya on the occasion of his 80th birthday

\begin{abstract}
We consider positive solutions $u$ of the semilinear biharmonic equation $\Delta^{2} u=|x|^{-\frac{n+4}{2}} g\left(|x|^{\frac{n-4}{2}} u\right)$ in $\mathbb{R}^{n} \backslash\{0\}$ with non-removable singularities at the origin. Under natural assumptions on the nonlinearity $g$, we show that $|x|^{\frac{n-4}{2}} u$ is a periodic function of $\ln |x|$ and we classify all such solutions.
\end{abstract}

\section{INTRODUCTION AND MAIN RESULTS}

We are interested in positive solutions $u$ of the semilinear biharmonic equation

$$
\Delta^{2} u=\frac{1}{|x|^{\frac{n+4}{2}}} g\left(|x|^{\frac{n-4}{2}} u\right) \quad \text { in } \mathbb{R}^{n} \backslash\{0\}
$$

which may have singularities at the origin. We always assume $n \geqslant 5$. The nonlinearity $g \in C^{1}\left(\mathbb{R}_{+}\right)$is taken to satisfy a set of growth conditions which will be specified below. The seemingly strange way the right hand side of equation (1) is written ensures invariance of the equation under rotations, dilations and inversion in the unit sphere. The form becomes very natural later when we pass to logarithmic radial coordinates. We will always interpret (II) in the weak sense, that is, we assume $u \in H_{\mathrm{loc}}^{2}\left(\mathbb{R}^{n} \backslash\{0\}\right)$, $g(u) \in L_{\mathrm{loc}}^{1}\left(\mathbb{R}^{n} \backslash\{0\}\right)$ and

$$
\int_{\mathbb{R}^{n}} \Delta u \Delta \varphi=\int_{\mathbb{R}^{n}} g(u) \varphi \quad \text { for all } \varphi \in C_{0}^{\infty}\left(\mathbb{R}^{n} \backslash\{0\}\right) .
$$

Our goal is to classify all positive solutions of (1). Under some natural assumptions on $g$ we will be able to show that, if $u$ has a non-removable singularity at 0 , then $|x|^{\frac{n-4}{2}} u$ is a periodic function of $\ln |x|$ and, up to dilations, all such functions are uniquely parametrized by the minimal value of this periodic function.

Date: March 7, 2019.

(C) 2019 by the authors. This paper may be reproduced, in its entirety, for non-commercial purposes.

Partial support through US National Science Foundation grant DMS-1363432 (R.L.F.) and Studienstiftung des deutschen Volkes (T.K.) is acknowledged. 
The simplest example of a nonlinearity to which our results apply is $g(t)=t^{\frac{n+4}{n-4}}$, where the equation becomes

$$
\Delta^{2} u=u^{\frac{n+4}{n-4}} \quad \text { in } \mathbb{R}^{n} \backslash\{0\} .
$$

Note that the exponent in this equation is critical in the sense of Sobolev's embedding theorem. We have treated equation (2) in our previous paper [3] and our goal now is to extend these results to a more general class of equations. In addition, we will be able to simplify some parts of the argument in [3].

Other equations that we can treat in this paper are, for instance,

$$
\Delta^{2} u=|x|^{-\frac{n+4}{2}+q \frac{n-4}{2}} u^{q} \quad \text { in } \mathbb{R}^{n} \backslash\{0\} .
$$

with $1<q \leqslant \frac{n+4}{n-4}$, as well as equations whose right hand side is equal to a finite sum, with positive coefficients, of terms as on the right side of (3). We can also allow for a 'Hardy-Rellich' term on the right side, that is,

$$
\Delta^{2} u=\beta|x|^{-4} u+|x|^{-\frac{n+4}{2}+q \frac{n-4}{2}} u^{q} \quad \text { in } \mathbb{R}^{n} \backslash\{0\}
$$

with $1<q \leqslant \frac{n+4}{n-4}$, provided the constant satisfies $0<\beta<\frac{n^{2}(n-4)^{2}}{16}$. Note that $\frac{n^{2}(n-4)^{2}}{16}$ is the sharp constant in the Hardy-Rellich inequality.

The precise assumptions on the nonlinearity $g$ are as follows,

$$
\begin{cases}g \in C^{1}\left(\mathbb{R}_{+}\right), g>0, \lim _{t \rightarrow 0} g(t)=0, & \\ \frac{g(t)}{t}<g^{\prime}(t) \leqslant \frac{n+4}{n-4} \frac{g(t)}{t} & \text { for all } t>0, \\ \beta:=\lim _{t \rightarrow 0} g^{\prime}(t)<\frac{n^{2}(n-4)^{2}}{16}, & \text { for all } t \geqslant 1,\end{cases}
$$

It is part of the assumptions on $g$ that the limits appearing in (5) exist.

Note that equations (3) and (4) correspond to the choices $g(t)=t^{q}$ and $g(t)=\beta t+t^{q}$, respectively. These functions clearly satisfy all the requirements in (5).

Let us discuss assumptions (5) for general functions $g$. The inequality $g^{\prime}(t) \leqslant \frac{n+4}{n-4} \frac{g(t)}{t}$ means that the non-linearity is subcritical or critical in the sense of Sobolev's embedding theorem, while the inequality $g(t) \geqslant c t^{q}$ is a superlinearity assumption for large values of the argument of $g$.

We proceed in two steps, the first one being a proof that positive solutions of (11) are radial and the second one being a careful analysis of the resulting ordinary differential equation.

The following is our first main result.

Theorem 1 (Radial symmetry). Suppose that g satisfies (5) and let $u \in H_{\mathrm{loc}}^{2}\left(\mathbb{R}^{n} \backslash\{0\}\right)$ be a solution of (1) with $u>0$. 
Assume that either $u \notin L^{\frac{2 n}{n-4}}\left(\mathbb{R}^{n}\right)$ or that the inequality $g^{\prime}(t) \leqslant \frac{n+4}{n-4} \frac{g(t)}{t}$ in (15) is strict for a.e. $t>0$.

Then $u$ is radially symmetric-decreasing with respect to 0 .

When $u \in L^{\frac{2 n}{n-4}}\left(\mathbb{R}^{n}\right)$ and the inequality $g^{\prime}(t) \leqslant \frac{n+4}{n-4} \frac{g(t)}{t}$ in (5) is not strict for a.e. $t>0$, then the conclusion of the theorem need not hold. Indeed, it is well-known that equation (1) with $g(t)=c t^{\frac{n+4}{n-4}}$, which is translation invariant, has a solution which is strictly radially symmetric-decreasing with respect to an arbitrary given point. This shows that some extra condition is needed to conclude radial symmetry with respect to the origin, although the condition given in the theorem can probably be relaxed.

Remark 2. We will show in Section 13 that, in fact, $\frac{\partial u}{\partial|x|}<0$ for all $x \in \mathbb{R}^{n} \backslash\{0\}$, using ODE methods.

We now proceed to the second step of our argument. According to Theorem 3 we can write equation (11) as an ordinary differential equation. It becomes particularly simple in logarithmic coordinates. That is, we make the so-called Emden-Fowler change of variables

$$
u(x)=|x|^{-\frac{n-4}{2}} v(\ln |x|) .
$$

After a short computation, we can write (11) as

$$
v^{(4)}-A v^{\prime \prime}+B v=g(v) \quad \text { in } \mathbb{R},
$$

with

$$
A=\frac{n(n-4)+8}{2} \quad \text { and } \quad B=\frac{n^{2}(n-4)^{2}}{16}
$$

The following is our second main result. Recall from (5) that by definition $\beta=$ $\lim _{t \rightarrow 0} g^{\prime}(t)$.

Theorem 3 (Classification of ODE solutions). Suppose that g satisfies (5).

Then any positive solution $v \in C^{4}(\mathbb{R})$ of (6) is either constant, or homoclinic to zero, or periodic. These solutions can be classified, up to translations, by their minimal value in the following sense.

(i) There is a unique $a_{0}>0$ such that $g\left(a_{0}\right)=B a_{0}$. Moreover, if $v \in C^{4}(\mathbb{R})$ is a positive solution of $(6)$, then $\inf _{\mathbb{R}} v \leqslant a_{0}$, with equality if and only if $v$ is a non-zero constant.

(ii) If $a \in\left(0, a_{0}\right)$, then there is a unique (up to translations) bounded solution $v \in C^{4}(\mathbb{R})$ of (6) with $\inf _{\mathbb{R}} v=a$. This solution is periodic, has a unique local maximum and minimum per period and is symmetric with respect to its local extrema. 
(iii) There is a unique (up to translations) positive solution $v \in C^{4}(\mathbb{R})$ of (6) with $\inf _{\mathbb{R}} v=0$. This solution is symmetric-decreasing and satisfies $v(t) \leqslant$ $C_{\epsilon} e^{-(\sqrt{\mu}-\epsilon)|t|}$ for any $\epsilon>0$, where $\mu=\frac{1}{2}\left(A-\sqrt{A^{2}-4(B-\beta)}\right)$. Moreover, if

$$
|g(t)-\beta t| \leqslant C t^{r} \quad \text { for all } t \leqslant 1, \quad \text { for some } r>1, C<\infty,
$$

then $\lim _{|t| \rightarrow \infty} e^{\sqrt{\mu}|t|} v(t)$ exists and is finite. When $g(t) \geqslant \beta t$ for all $t>0$, then the limit is positive.

Note that for homoclinic solutions we prove exponential decay, since the assumption $\beta<B$ implies $\mu>0$. Moreover, recalling the explicit expression of $A$ and $B$, we obtain

$$
\sqrt{\mu}=\frac{n-4}{2} \quad \text { if } \beta=0
$$

The combination of Theorems 1 and 3 yields immediately the following classification of positive singular solutions of the PDE (11). To state this result, we denote, for $a \in\left[0, a_{0}\right]$, by $v_{a}$ the unique positive solution to (6) obtained from Theorem 3 by requiring that $\inf _{\mathbb{R}} v_{a}=a$ and $v_{a}(0)=\max _{\mathbb{R}} v$. For $a \in\left(0, a_{0}\right)$ we denote by $L_{a}$ the minimal period length of $v_{a}$ and we set $L_{a_{0}}=0$ and $L_{0}=\infty$.

Theorem 4 (Classification of PDE solutions). Suppose that $g$ satisfies (5). Let $u \in$ $C^{4}\left(\mathbb{R}^{n} \backslash\{0\}\right)$ be a positive solution of (1) and assume that either $u \notin L^{\frac{2 n}{n-4}}\left(\mathbb{R}^{n}\right)$ or that the inequality $g^{\prime}(t) \leqslant \frac{n+4}{n-4} \frac{g(t)}{t}$ in (15) is strict for a.e. $t>0$.

Then there are $a \in\left[0, a_{0}\right]$ and $L \in\left[0, L_{a}\right]$ such that

$$
u(x)=|x|^{-\frac{n-4}{2}} v_{a}(\log |x|+L),
$$

where $v_{a}$ is the solution of (6) introduced above.

In particular, if $a=a_{0}$, then $u(x)=|x|^{-\frac{n-4}{2}} a_{0}$, and if $a \in\left(0, a_{0}\right)$, then

$$
\begin{aligned}
0 & <\liminf _{|x| \rightarrow 0}|x|^{\frac{n-4}{2}} u(x)=\liminf _{|x| \rightarrow \infty}|x|^{\frac{n-4}{2}} u(x) \\
& <\limsup _{|x| \rightarrow 0}|x|^{\frac{n-4}{2}} u(x)=\limsup _{|x| \rightarrow \infty}|x|^{\frac{n-4}{2}} u(x)<\infty .
\end{aligned}
$$

Under assumption (7), if $a=0$, then

$$
\lim _{|x| \rightarrow 0}|x|^{\frac{n-4}{2}-\mu} u(x)=\lim _{|x| \rightarrow \infty}|x|^{\frac{n-4}{2}+\mu} u(x)<\infty
$$

and, if $g(t) \geqslant \beta t$ for all $t>0$, then the limit is positive.

Let us discuss the implications of this theorem to the question of removability of singularities. When $a>0$, the solution $u$ has a non-removable singularity at the origin. When $a=0$, the situation depends on whether the parameter $\beta$ from (5) vanishes or not. For $\beta=0$, when $\mu=\frac{n-4}{2}$, the solution extends continuously to the 
origin, while for $\beta>0$, when $\mu<\frac{n-4}{2}$, the solution has a power-like singularity at the origin. Note, however, that this singularity is weaker than in the case $a>0$.

It is also remarkable that the behavior near the origin is closely related to the behavior of $u$ at infinity.

In the remainder of this paper we prove Theorem 1, Remark 2 and Theorem 3. The first one is proved in Section 2 using the method of moving planes, while the second and third one are proved in Section 3 using ODE techniques.

\section{Method of moving Planes}

Our goal in this section is to prove Theorem 1. We will deduce it from the following theorem, which is our main symmetry result. We point out that for the proof of Theorem 5 below, we actually do not need the lower bound $g(t) / t<g^{\prime}(t)$ from (5).

Throughout the following, we will fix a point $a \in \mathbb{R}^{n} \backslash\{0\}$ and consider

$$
S=\{0, a\} \subset \mathbb{R}^{n} .
$$

We shall prove

Theorem 5. Suppose that $g$ satisfies (5).

Let $k$ be a positive function in $\mathbb{R}^{n} \backslash S$ which is symmetric-decreasing with respect to a hyperplane $H$ passing through 0 and $a$. Assume that $k(x) \gtrsim \operatorname{dist}(x, S)^{-1}$ in a neighborhood of $S$, that $k \in L_{\mathrm{loc}}^{(n+2) / 4}\left(\mathbb{R}^{n}\right)$ and that $k \in L^{n}(\Omega)$ for every $\Omega$ which is a positive distance away from $S$.

Let $v \in H_{\mathrm{loc}}^{2}\left(\mathbb{R}^{n} \backslash S\right)$ be a weak solution of

$$
\Delta^{2} v=k(x)^{\frac{n+4}{2}} g\left(k(x)^{-\frac{n-4}{2}} v(x)\right) \quad \text { in } \mathbb{R}^{n} \backslash S
$$

and assume that $v>0$ and that $v \in L^{\frac{2 n}{n-4}}(\Omega)$ for every $\Omega$ which is a positive distance away from $S$.

Assume that either $v \notin L^{\frac{2 n}{n-4}}\left(\mathbb{R}^{n}\right)$, or that the inequality $g^{\prime}(t) \leqslant \frac{n+4}{n-4} \frac{g(t)}{t}$ in (15) is strict for a.e. $t>0$ and $k$ is strictly symmetric-decreasing.

Then $v$ is strictly symmetric-decreasing with respect to $H$.

By saying that a function $f$ is symmetric-decreasing with respect to a hyperplane $H$ through 0 with normal vector $e$ we mean that

$$
f(y-t e)=f(y+t e) \geqslant f(y+s e) \quad \text { for all } 0 \leqslant s \leqslant t \text { and all } y \cdot e=0 .
$$

By saying that $f$ is strictly symmetric-decreasing we mean that the inequality is strict for $s<t$. 
2.1. Kelvin transformation and proof of Theorem 1, Although neither $u$ nor $|x|^{-1}$ from Theorem 11 satisfy the assumptions of Theorem 5, we will now show that we can transform equation (11) to another equation for which these assumptions are satisfied. Indeed, for $z \neq 0$ we denote by $z^{*}=\frac{z}{|z|^{2}}$ the inversion of $z$ about the unit sphere and for any function $u$ on $\mathbb{R}^{n} \backslash\{0\}$, we define its Kelvin-type transform $u_{z}^{*}$ with respect to the point $z$ by

$$
u_{z}^{*}(x):=\frac{1}{|x|^{n-4}} u\left(\frac{x}{|x|^{2}}+z\right), \quad x \in \mathbb{R}^{n} \backslash\left\{0,-z^{*}\right\} .
$$

If $z=0$, we denote $u^{*}:=u_{0}^{*}$.

The technique of improving the decay properties of a solution to a conformally invariant equation by passing to the Kelvin transform goes back to [2] and has been widely used in the context of the method of moving planes. Since our equation (11) is, in general, not translation invariant, $u_{z}^{*}$ with $z \neq 0$ is, in general, no longer a solution of (1). It will, however, satisfy a related equation. Indeed, if $u$ solves (11), then from the formula

$$
\Delta^{2} \varphi^{*}(x)=\frac{1}{|x|^{n+4}} \Delta^{2} \varphi\left(\frac{x}{|x|^{2}}\right), \quad \varphi \in C_{0}^{\infty}\left(\mathbb{R}^{n} \backslash\{0\}\right)
$$

and a straightforward calculation using the fact that $\left|\frac{x}{|x|^{2}}-\frac{z}{|z|^{2}}\right|=\frac{|x-z|}{|x||z|}$, it follows that $u_{z}^{*}$ satisfies the equation

$$
\Delta^{2} u_{z}^{*}(x)=k_{z}(x)^{\frac{n+4}{2}} g\left(k_{z}(x)^{-\frac{n-4}{2}} u_{z}^{*}(x)\right) \text { in } \mathbb{R}^{n} \backslash\left\{0,-z^{*}\right\}
$$

with

$$
k_{z}(x)=\frac{\left|z^{*}\right|}{|x|\left|x+z^{*}\right|}, \quad x \in \mathbb{R}^{n} \backslash\left\{0,-z^{*}\right\},
$$

for $z \neq 0$ and $k_{0}(x)=|x|^{-1}$ if $z=0$. This equation is understood in the weak sense, i.e.

$\int_{\mathbb{R}^{n}} \Delta u_{z}^{*} \Delta \varphi=\int_{\mathbb{R}^{n}} k_{z}(x)^{\frac{n+4}{2}} g\left(k_{z}(x)^{-\frac{n-4}{2}} u_{z}^{*}(x)\right) \varphi(x) \mathrm{d} x \quad$ for all $\varphi \in C_{0}^{\infty}\left(\mathbb{R}^{n} \backslash\left\{0,-z^{*}\right\}\right)$.

Notice that this means that if $u$ solves equation (11), then so does $u^{*}$.

Here are some more properties of $u_{z}^{*}$ which we need for our argument.

Lemma 6. Suppose that $u \in H_{\mathrm{loc}}^{2}\left(\mathbb{R}^{n} \backslash\{0\}\right)$.

(a) If $z \neq 0$, then $u_{z}^{*} \in H_{\mathrm{loc}}^{2}\left(\mathbb{R}^{n} \backslash\left\{0,-z^{*}\right\}\right)$ and, in fact, we have

$$
\int_{\Omega}\left|\Delta u_{z}^{*}\right|^{2}<\infty \quad \text { and } \quad \int_{\Omega}\left|u_{z}^{*}\right|^{\frac{2 n}{n-4}}<\infty
$$

for every $\Omega$ which is a positive distance away from 0 and $-z^{*}$.

(b) If $z=0$, then $u^{*} \in H_{\mathrm{loc}}^{2}\left(\mathbb{R}^{n} \backslash\{0\}\right)$. 
Proof. Let $\Omega \subset \mathbb{R}^{n}$ be a positive distance away from 0 and $-z^{*}$ and let $\Omega^{*}=\left\{x^{*}\right.$ : $x \in \Omega\}$. Then the set $\Omega^{*}+z$ is bounded and a positive distance away from 0 . By a change of variables, we have

$$
\int_{\Omega} u_{z}^{* \frac{2 n}{n-4}}(x) \mathrm{d} x=\int_{\Omega^{*}} u^{\frac{2 n}{n-4}}(x+z) \mathrm{d} x=\int_{\Omega^{*}+z} u^{\frac{2 n}{n-4}}<\infty
$$

since $u \in L_{\text {loc }}^{\frac{2 n}{n-4}}\left(\mathbb{R}^{n} \backslash\{0\}\right)$ by Sobolev embedding.

Let us now turn to the second derivative. As in [8, Proof of Lemma 3.6], using Kelvin's transformation rule

$$
\Delta\left(|x|^{2-n} u\left(\frac{x}{|x|^{2}}\right)\right)=|x|^{-n-2}(\Delta u)\left(\frac{x}{|x|^{2}}\right),
$$

we have by the product rule for Sobolev functions that $\Delta u_{z}^{*}$ exists weakly in $\mathbb{R}^{n} \backslash\left\{0,-z^{*}\right\}$ and is given by

$$
\begin{aligned}
& \Delta u_{z}^{*}(x)=\Delta\left(|x|^{2}|x|^{2-n} u\left(\frac{x}{|x|^{2}}+z\right)\right) \\
& =2 n|x|^{2-n} u\left(\frac{x}{|x|^{2}}+z\right)+4 x \cdot \nabla\left(|x|^{2-n} u\left(\frac{x}{|x|^{2}}+z\right)\right)+|x|^{-n} \Delta u\left(\frac{x}{|x|^{2}}+z\right) \\
& =-2(n-4)|x|^{2-n} u\left(\frac{x}{|x|^{2}}+z\right)+4|x|^{2-n} x \cdot \nabla\left(u\left(\frac{x}{|x|^{2}}+z\right)\right)+|x|^{-n} \Delta u\left(\frac{x}{|x|^{2}}+z\right) .
\end{aligned}
$$

We show square integrability of each of the three terms on the right side. First,

$$
\left.\left.\int_{\Omega}|| x\right|^{2-n} u\left(\frac{x}{|x|^{2}}+z\right)\right|^{2}=\int_{\Omega^{*}}|x|^{-4}|u(x+z)|^{2} \lesssim \int_{\widetilde{\Omega^{*}}}|\Delta u(x+z)|^{2}<\infty
$$

by the Hardy-Rellich inequality, where we choose some larger $\widetilde{\Omega^{*}} \supset \overline{\Omega^{*}}$ which is still a positive distance away from $-z$. This follows by a simple argument using cutoff functions. For the second term of (12), we have $\left|\nabla\left(u\left(\frac{x}{|x|^{2}}+z\right)\right)\right| \lesssim|x|^{-2}\left|\nabla u\left(\frac{x}{|x|^{2}}+z\right)\right|$ and therefore

$$
\begin{aligned}
\left.\left.\int_{\Omega}|| x\right|^{2-n} x \cdot \nabla\left(u\left(\frac{x}{|x|^{2}}+z\right)\right)\right|^{2} & \lesssim \int_{\Omega}|x|^{2-2 n}\left|\nabla u\left(\frac{x}{|x|^{2}}+z\right)\right|^{2}=\int_{\Omega}|x|^{-2}|\nabla u(x+z)|^{2} \\
& \lesssim\|u\|_{H^{2}\left(\widetilde{\Omega^{*}}+z\right)}^{2}<\infty
\end{aligned}
$$

by Hardy's inequality applied to $\partial_{j} u, j=1, \ldots, n$, with some set $\widetilde{\Omega^{*}}$ as above. The third term of (12) gives

$$
\int_{\Omega}|x|^{-2 n}\left|\Delta u\left(\frac{x}{|x|^{2}}+z\right)\right|^{2}=\int_{\Omega^{*}+z}|\Delta u(x)|^{2}<\infty
$$

because $u \in H_{\text {loc }}^{2}\left(\mathbb{R}^{n} \backslash\{0\}\right)$.

The proof of part (b) is similar, but simpler, and we omit it.

We can now deduce Theorem 1 from Theorem 5 in a straightforward way. 
Proof of Theorem 1 given Theorem 5. Step 1. Let $z \neq 0$ and denote by $l_{z}$ the line through 0 and $z$. We shall show that for any hyperplane $H$ orthogonal to $l_{z}$ the function $u_{z}^{*}$ is strictly radially symmetric-decreasing in $H$ with respect to $H \cap l_{z}$.

We want to deduce this from Theorem 5 with $a=-z^{*}, v=u_{z}^{*}$ and $k=k_{z}$ applied to equation (10). Let us verify that the assumptions of this theorem are satisfied.

This is clear for the assumptions on $g$. Next, the function $k(x)=\frac{\left|z^{*}\right|}{|x|\left|x+z^{*}\right|}$ is strictly symmetric-decreasing with respect to any hyperplane passing through 0 and $z$ (which is the same as passing through 0 and $a=-z^{*}$ ). It decreases as $|x|^{-2}$ as $|x| \rightarrow \infty$ and behaves as $\operatorname{dist}(x, S)^{-1}$ near $S$. Therefore the assumptions on $k$ are satisfied. Finally, according to Lemma [6 the function $v=u_{z}^{*}$ belongs to $H_{\mathrm{loc}}^{2}\left(\mathbb{R}^{n} \backslash\left\{0,-z^{*}\right\}\right)$ and is in $L^{\frac{2 n}{n-4}}$ on any $\Omega$ which is a positive distance away from 0 and $-z^{*}$. As in the proof of that lemma one sees that $u \in L^{\frac{2 n}{n-4}}\left(\mathbb{R}^{n}\right)$ if and only if $u_{z}^{*} \in L^{\frac{2 n}{n-4}}\left(\mathbb{R}^{n}\right)$.

Thus, from Theorem 5 we deduce that $u_{z}^{*}$ is strictly symmetric-decreasing with respect to any hyperplane passing through 0 and $z$. This implies the assertion.

Step 2. We now deduce that $u$ is radially symmetric-decreasing.

By letting $z \rightarrow 0$ along a fixed direction $\nu \in \mathbb{S}^{n-1}$, the assertion of Step 1 implies that in any hyperplane with normal $\nu, u^{*}$ is radially symmetric-decreasing with respect to the point where the hyperplane intersects the line through 0 in direction $\nu$. Since $\nu$ is arbitrary, we conclude that $u^{*}$ is radially symmetric-decreasing with respect to 0 .

Applying what we have proved so far to $u^{*}$, which, by Lemma 6 , is in $H_{\text {loc }}^{2}\left(\mathbb{R}^{n} \backslash\{0\}\right)$ if and only if $u$ is and which solves (11) if and only if $u$ does, we also find that $u=\left(u^{*}\right)^{*}$ is radially symmetric-decreasing with respect to 0 .

2.2. Some integrability estimates. We have therefore reduced the proof of Theorem 1 to the proof of Theorem 5 .

In this and the next subsection we always assume that $g$ and $v$ satisfy the assumptions in Theorem [5] and that (8) holds in the weak sense.

Our first step in proving Theorem 5 is to understand the behavior of the solution near the singular points. This will later allow us to use a larger class of test functions, including functions whose support contains $S$. This amounts to proving integrability of $v$ at $S$ and constitutes the purpose of the present subsection. Our arguments in this subsection are inspired by [9, Lemmas 3.1 and 3.2].

Lemma 7. We have $k^{\frac{n+4}{2}} g\left(k^{-\frac{n-4}{2}} v\right) \in L_{\mathrm{loc}}^{1}\left(\mathbb{R}^{n}\right)$ and $k^{\frac{n+4}{2}} g\left(k^{-\frac{n-4}{2}} v\right) \in L^{\frac{2 n}{n+4}}(\Omega)$ for every $\Omega$ which is a positive distance away from $S$. 
Proof. First, assume that $\Omega$ is a positive distance away from $S$. The inequality $g^{\prime}(t) \leqslant$ $\frac{n+4}{n-4} \frac{g(t)}{t}$ in (5) implies by integration that $g(t) \leqslant g(1) t^{(n+4) /(n-4)}$ for $t \geqslant 1$ and therefore

$$
g(t) \leqslant g(1) t^{(n+4) /(n-4)}+\sup _{0 \leqslant t \leqslant 1} g \lesssim t^{(n+4) /(n-4)}+1 \quad \text { for all } t \geqslant 0 .
$$

Thus, $k^{\frac{n+4}{2}} g\left(k^{-\frac{n-4}{2}} v\right) \lesssim v^{\frac{n+4}{n-4}}+k^{\frac{n+4}{2}}$ and consequently

$$
\int_{\Omega}\left(k^{\frac{n+4}{2}} g\left(k^{-\frac{n-4}{2}} v\right)\right)^{\frac{2 n}{n+4}} \lesssim \int_{\Omega}\left(v^{\frac{2 n}{n-4}}+k^{n}\right) .
$$

The integral on the right side is finite by our assumptions.

In order to prove the local integrability, let $\eta$ be a smooth, non-negative function on $\mathbb{R}^{n}$ with $\eta \equiv 1$ near 0 and with support in a ball not containing $a$. We shall show that $\int k^{\frac{n+4}{2}} g\left(k^{-\frac{n-4}{2}} v\right) \eta<\infty$. This and a corresponding assertion for $\eta$ supported near $a$ proves the lemma.

For $\epsilon>0$ so small that $\eta \equiv 1$ on $\{|x| \leqslant 2 \epsilon\}$, we can find non-negative cut-off functions $\eta_{\epsilon} \in C_{0}^{\infty}$ such that

$$
\eta_{\epsilon}(x) \begin{cases}\equiv 0 & \text { if }|x| \leqslant \epsilon \\ \equiv \eta & \text { if }|x| \geqslant 2 \epsilon\end{cases}
$$

and

$$
\left|D^{k} \eta_{\epsilon}(x)\right| \lesssim \epsilon^{-k} \text { for all } k=1,2,3,4
$$

For $q$ as in assumption (5), let $m=\frac{4 q}{q-1}$ and define $\xi_{\epsilon}=\left(\eta_{\epsilon}\right)^{m}$. Since $\xi_{\epsilon}$ is supported away from $S$, it is a valid test function for equation (8), and we obtain

$$
\int_{\mathbb{R}^{n}} k^{\frac{n+4}{2}} g\left(k^{-\frac{n-4}{2}} v\right) \xi_{\epsilon}=\int_{\mathbb{R}^{n}} \Delta v \Delta \xi_{\epsilon}=\int_{\mathbb{R}^{n}} v \Delta^{2} \xi_{\epsilon} .
$$

Observing that

$$
\left|\Delta^{2} \xi_{\epsilon}\right| \lesssim \epsilon^{-4} \eta_{\epsilon}^{m-4} 1_{\{\epsilon<|x|<2 \epsilon\}}+\Delta^{2} \eta^{m}=\epsilon^{-4} \xi_{\epsilon}^{1 / q} 1_{\{\epsilon<|x|<2 \epsilon\}}+\Delta^{2} \eta^{m},
$$

and using the assumed lower bound on $k$ (note that $q \leqslant \frac{n+4}{n-4}$, since $g(t) \lesssim t^{(n+4) /(n-4)}$ ), we find that

$$
\begin{aligned}
\int_{\mathbb{R}^{n}} k^{\frac{n+4}{2}} g\left(k^{-\frac{n-4}{2}} v\right) \xi_{\epsilon} & \lesssim \epsilon^{-4} \int_{\{\epsilon<|x|<2 \epsilon\}} v \xi_{\epsilon}^{1 / q}+\int v \Delta^{2} \eta^{m} \\
& \lesssim \epsilon^{-4+\frac{n+4}{2 q}-\frac{n-4}{2}} \int_{\{\epsilon<|x|<2 \epsilon\}} v k^{-\frac{n-4}{2}+\frac{n+4}{2 q}} \xi_{\epsilon}^{1 / q}+\int v \Delta^{2} \eta^{m} \\
& \lesssim \epsilon^{-4+\frac{n+4}{2 q}-\frac{n-4}{2}+n \frac{q-1}{q}}\left(\int_{\{\epsilon<|x|<2 \epsilon\}} v^{q} k^{-q \frac{n-4}{2}+\frac{n+4}{2}} \xi_{\epsilon}\right)^{\frac{1}{q}}+\int v \Delta^{2} \eta^{m}
\end{aligned}
$$

Because of the equivalence

$$
-4+\frac{n+4}{2 q}-\frac{n-4}{2}+n \frac{q-1}{q}>0 \quad \Leftrightarrow \quad q>1,
$$


the exponent of $\epsilon$ is positive and therefore we can drop the factor in front of the first term. The second term is finite since $\Delta^{2} \eta^{m}=0$ near zero, and independent of $\epsilon$. To close the estimate, we will use the fact that

$$
t^{q} \leqslant c^{-1} g(t)+1 \lesssim g(t)+1 \text { for all } t \geqslant 0
$$

Using this, we can estimate

$$
\begin{aligned}
& \left(\int_{\{\epsilon<|x|<2 \epsilon\}} v^{q} k^{-q \frac{n-4}{2}+\frac{n+4}{2}} \xi_{\epsilon}\right)^{\frac{1}{q}} \\
\lesssim & \left(\int_{\{\epsilon<|x|<2 \epsilon\}} k^{\frac{n+4}{2}} g\left(k^{-\frac{n-4}{2}} v\right) \xi_{\epsilon}+\int_{\{\epsilon<|x|<2 \epsilon\}} k^{\frac{n+4}{2}}\right)^{\frac{1}{q}} .
\end{aligned}
$$

The second term on the right side is finite by assumption. We have thus proved that

$$
\int_{\mathbb{R}^{n}} k^{\frac{n+4}{2}} g\left(k^{-\frac{n-4}{2}} v\right) \xi_{\epsilon} \lesssim\left(\int_{\mathbb{R}^{n}} k^{\frac{n+4}{2}} g\left(k^{-\frac{n-4}{2}} v\right) \xi_{\epsilon}\right)^{\frac{1}{q}}+1,
$$

which implies that

$$
\int_{\mathbb{R}^{n}} k^{\frac{n+4}{2}} g\left(k^{-\frac{n-4}{2}} v\right) \xi_{\epsilon} \lesssim 1
$$

Letting $\epsilon \rightarrow 0$, we conclude by monotone convergence that

$$
\int_{\mathbb{R}^{n}} k^{\frac{n+4}{2}} g\left(k^{-\frac{n-4}{2}} v\right) \eta<\infty
$$

which finishes the proof.

We can use the fundamental integrability properties from Lemma 7 to enlarge the class of functions one can test equation (8) against. This is the content of the next lemma.

Lemma 8. Let $\varphi \in \dot{H}^{2}\left(\mathbb{R}^{n}\right)$ and assume, in addition, that $\Delta \varphi \equiv 0$ in a neighborhood of $S$. Then

$$
\int_{\mathbb{R}^{n}} \Delta v \Delta \varphi=\int_{\mathbb{R}^{n}} k^{\frac{n+4}{2}} g\left(k^{-\frac{n-4}{2}} v\right) \varphi
$$

By definition, $\dot{H}^{2}\left(\mathbb{R}^{n}\right)$ is the completion of $C_{0}^{\infty}\left(\mathbb{R}^{n}\right)$ with respect to $\|\Delta u\|_{2}$.

Proof. We begin by localizing the problem. We choose non-negative $C^{\infty}$ functions $\chi_{0}$, $\chi_{a}$ and $\chi_{\infty}$ such that $\chi_{0}+\chi_{a}+\chi_{\infty} \equiv 1$ on $\mathbb{R}^{n}$ and such that $\chi_{0}$ and $\chi_{a}$ are $\equiv 1$ near the points 0 and $a$, respectively, and both have compact support.

Given $\varphi$ as in the lemma, it clearly suffices to prove the theorem for each of the functions $\chi_{0} \varphi, \chi_{a} \varphi$ and $\chi_{\infty} \varphi$. Note that all three functions belong to $\dot{H}^{2}\left(\mathbb{R}^{n}\right)$ and are harmonic near $S$.

The identity for $\chi_{\infty} \varphi$ follows by a straightforward approximation argument using the fact that on the support of $\chi_{\infty} \varphi, \Delta v$ is in $L^{2}$ and, by Lemma $7, k^{\frac{n+4}{2}} g\left(k^{-\frac{n-4}{2}} v\right)$ is in $L^{\frac{2 n}{n+4}}$. 
The argument for $\chi_{a} \varphi$ is the same as that for $\chi_{0} \varphi$, so we focus on the latter. To ease notation, we write $\varphi$ instead of $\chi_{0} \varphi$ and assume that $\varphi$ has compact support not containing $a$ and is harmonic near 0 . By harmonicity, $\varphi$ is $C^{\infty}$ near zero and, in particular, $\varphi$ and all its derivatives are bounded in a ball $B$ near zero.

For $\epsilon>0$ so small that $B(0,2 \epsilon) \subset B$, fix $\eta_{\epsilon} \in C^{\infty}\left(\mathbb{R}^{n}\right)$ such that

$$
\eta_{\epsilon}(x) \begin{cases}\equiv 0 & \text { if }|x| \leqslant \epsilon \\ \equiv 1 & \text { if }|x| \geqslant 2 \epsilon\end{cases}
$$

and $\left|D^{k} \eta_{\epsilon}\right| \lesssim \epsilon^{-k}$ for $k=1,2,3,4$. We can now test the equation for $v$ with $\varphi \eta_{\epsilon}$, which is a valid test function, since it is in $\dot{H}^{2}\left(\mathbb{R}^{n}\right)$ and supported away from $S$. We obtain

$$
\int_{\mathbb{R}^{n}} \Delta v \Delta\left(\varphi \eta_{\epsilon}\right)=\int_{\mathbb{R}^{n}} k^{\frac{n+4}{2}} g\left(k^{-\frac{n-4}{2}} v\right) \varphi \eta_{\epsilon} .
$$

As $\epsilon \rightarrow 0$, the right hand side tends to $\int_{\mathbb{R}^{n}} k^{\frac{n+4}{2}} g\left(k^{-\frac{n-4}{2}} v\right) \varphi$ by dominated convergence, using $\varphi \in L^{\infty}(B)$ and Lemma 7. To evaluate the left hand side, we write

$$
\Delta\left(\varphi \eta_{\epsilon}\right)=\eta_{\epsilon} \Delta \varphi+2 \nabla \varphi \cdot \nabla \eta_{\epsilon}+\varphi \Delta \eta_{\epsilon}
$$

and consider the three terms of (15) separately. For the first term, since $\Delta \varphi=0$ in $\left\{\eta_{\epsilon} \neq 1\right\}$, we have

$$
\int_{\mathbb{R}^{n}}(\Delta v \Delta \varphi) \eta_{\epsilon}=\int_{\mathbb{R}^{n}} \Delta v \Delta \varphi
$$

Therefore, to finish the proof, it remains to show that

$$
\lim _{\epsilon \rightarrow 0} \int_{\mathbb{R}^{n}} \Delta v\left(2 \nabla \varphi \cdot \nabla \eta_{\epsilon}+\varphi \Delta \eta_{\epsilon}\right)=0 .
$$

Using the facts that $\varphi \in \dot{H}^{2}\left(\mathbb{R}^{n}\right)$ and that $\Delta \varphi \equiv 0$ on $\operatorname{supp}\left(\nabla \eta_{\epsilon}\right) \cup \operatorname{supp}\left(\Delta \eta_{\epsilon}\right) \subset\{\epsilon<$ $|x|<2 \epsilon\} \subset B$, we obtain from integration by parts that

$$
\int_{\mathbb{R}^{n}} \Delta v\left(2 \nabla \varphi \cdot \nabla \eta_{\epsilon}+\varphi \Delta \eta_{\epsilon}\right)=\int_{\{\epsilon<|x|<2 \epsilon\}} v\left(\left(4 \sum_{i, j=1}^{n} \partial_{i j} \varphi \partial_{i j} \eta_{\epsilon}\right)+4 \nabla \varphi \cdot \nabla \Delta \eta_{\epsilon}+\varphi \Delta^{2} \eta_{\epsilon}\right) .
$$

Similarly to the proof of Lemma 7, since $k \gtrsim \epsilon^{-1}$ on $\{\epsilon<|x|<2 \epsilon\}$ and since $\partial_{i j} \varphi$ is bounded on $\{\epsilon<|x|<2 \epsilon\}$, we have

$$
\begin{aligned}
& \left|\int_{\{\epsilon<|x|<2 \epsilon\}} v \sum_{i, j=1}^{n} \partial_{i j} \varphi \partial_{i j} \eta_{\epsilon}\right| \\
& \lesssim \epsilon^{-\frac{n-4}{2}+\frac{n+4}{2 q}}\left(\int_{\{\epsilon<|x|<2 \epsilon\}} k^{\frac{n+4}{2}}\left(1+g\left(k^{-\frac{n-4}{2}} v\right)\right)\right)^{\frac{1}{q}}\left(\int_{\{\epsilon<|x|<2 \epsilon\}} \sum_{i, j=1}^{n}\left|\partial_{i j} \eta_{\epsilon}\right|^{\frac{q}{q-1}}\right)^{\frac{q-1}{q}}
\end{aligned}
$$

by Hölder's inequality. From Lemma 7 and the bound $\left|D^{2} \eta_{\epsilon}\right| \lesssim \epsilon^{-2}$, we infer that

$$
\left|\int_{\{\epsilon<|x|<2 \epsilon\}} v \sum_{i, j=1}^{n} \partial_{i j} \varphi \partial_{i j} \eta_{\epsilon}\right| \lesssim \epsilon^{-2+\frac{n(q-1)}{q}-\frac{n-4}{2}+\frac{n+4}{2 q}}
$$


By (13), we have $-2+\frac{n(q-1)}{q}-\frac{n-4}{2}+\frac{n+4}{2 q}>0$ and we conclude that

$$
\lim _{\epsilon \rightarrow 0} \int_{\mathbb{R}^{n}} v \sum_{i, j=1}^{n} \partial_{i j} \varphi \partial_{i j} \eta_{\epsilon}=0 .
$$

By an analogous argument, using boundedness of $\nabla \varphi$ and $\varphi$ on $B$ and the bounds $\left|D^{k} \eta_{\epsilon}\right| \leqslant \epsilon^{-k}$ for $k=3,4$, one can establish that

$$
\lim _{\epsilon \rightarrow 0} \int_{\mathbb{R}^{n}} v\left(\nabla \varphi \cdot \nabla \Delta \eta_{\epsilon}\right)=\lim _{\epsilon \rightarrow 0} \int_{\mathbb{R}^{n}} v \varphi \Delta^{2} \eta_{\epsilon}=0 .
$$

The proof of (16), and therefore of Lemma 8, is complete.

2.3. The method of moving planes. In this subsection we prove Theorem 5. Again we assume that $g$ and $v$ satisfy the assumptions of that theorem and that (8) holds in the weak sense.

Since the assumptions and the conclusion of the theorem are invariant under rotations, we may assume that $a_{1}=0$ and $H=\left\{x_{1}=0\right\}$.

For any number $\lambda<0$, we introduce the usual moving planes notation by letting $\Sigma_{\lambda}=\left\{x_{1}>\lambda\right\}, x^{\lambda}=\left(2 \lambda-x_{1}, x_{2}, \ldots, x_{n}\right), v_{\lambda}(x)=v\left(x^{\lambda}\right)$ and $k_{\lambda}(x)=k\left(x^{\lambda}\right)$. (This should not be confused with the function $k_{z}$ from Subsection 2.1.) Moreover, on $\Sigma_{\lambda}$, we define the difference function $w^{(\lambda)}=v-v_{\lambda}$. We will consider this function only in the half-space $\Sigma_{\lambda}$. When $\lambda$ is understood, we will often abbreviate this function by $w$.

The following lemma will be at the core of the moving planes argument used to prove Theorem 5 .

Lemma 9. Let $v, g$ and $k$ fulfill the assumptions of Theorem 5 and let $w^{(\lambda)}=v-v_{\lambda}$ on $\Sigma_{\lambda}$. Define, for $x \in \Sigma_{\lambda}$,

$$
V^{(\lambda)}(x)=k_{\lambda}(x)^{4} \frac{g\left(v(x) k_{\lambda}(x)^{-\frac{n-4}{2}}\right)-g\left(v_{\lambda}(x) k_{\lambda}(x)^{-\frac{n-4}{2}}\right)}{v(x) k_{\lambda}(x)^{-\frac{n-4}{2}}-v_{\lambda}(x) k_{\lambda}(x)^{-\frac{n-4}{2}}} .
$$

Then the following holds.

(a) We have $V^{(\lambda)} \geqslant 0$ and $V^{(\lambda)} \in L^{\frac{n}{4}}\left(\left\{w^{(\lambda)}<0\right\}\right)$ for all $\lambda<0$. Moreover,

$$
\lim _{\lambda \rightarrow-\infty} \int_{\{w(\lambda)<0\}}\left(V^{(\lambda)}\right)^{\frac{n}{4}}=0 .
$$

(b) Let $\psi \in \dot{H}^{2}\left(\Sigma_{\lambda}\right) \cap \dot{W}_{0}^{1, \frac{2 n}{n-2}}\left(\Sigma_{\lambda}\right)$ with $\psi \geqslant 0$ and assume that $\Delta \psi \equiv 0$ in a neighborhood of $S$. Then

$$
\int_{\Sigma_{\lambda}} \Delta w^{(\lambda)} \Delta \psi \geqslant-\int_{\Sigma_{\lambda}} V^{(\lambda)}\left(w^{(\lambda)}\right)_{-} \psi
$$


By definition, $\dot{W}_{0}^{1, \frac{2 n}{n-2}}\left(\Sigma_{\lambda}\right)$ is the completion of $C_{0}^{\infty}\left(\Sigma_{\lambda}\right)$ with respect to $\|\nabla u\|_{\frac{2 n}{n-2}}$.

Proof. As a rule, we will abbreviate $w=w^{(\lambda)}$ and $V=V^{(\lambda)}$.

Proof of (a). Firstly, since $k>0$ and $g^{\prime} \geqslant 0$ by (5), we have $V \geqslant 0$.

Secondly, as we have seen in the proof of Lemma 7, $g(t) \lesssim t^{\frac{n+4}{n-4}}$ for $t \geqslant 1$. Reinserting this bound into the assumed upper bound on $g^{\prime}$ and using the fact that $g^{\prime}$ is bounded on $(0,1]$ ( since $\lim _{t \rightarrow 0} g^{\prime}(t)$ exists and is finite), we obtain the bound

$$
g^{\prime}(t) \lesssim 1+t^{\frac{8}{n-4}}
$$

Therefore, by the mean value theorem,

$$
\frac{g(t)-g(s)}{t-s} \lesssim 1+t^{\frac{8}{n-4}} \quad \text { for all } 0<s<t .
$$

Applying (19) with $t=v_{\lambda}(x) k_{\lambda}(x)^{-\frac{n-4}{2}}$ and $s=v(x) k_{\lambda}(x)^{-\frac{n-4}{2}}$ and noticing that $t>s$ whenever $w<0$, we can bound

$$
\int_{\{w<0\}} V^{\frac{n}{4}} \lesssim \int_{\{w<0\}} k_{\lambda}^{n}+\int_{\{w<0\}} v_{\lambda}^{\frac{2 n}{n-4}} \leqslant \int_{\left\{x_{1}<\lambda\right\}}\left(k^{n}+v^{\frac{2 n}{n-4}}\right) .
$$

The right side is finite by the integrability assumptions on $k$ and $v$ and, by dominated convergence, tends to zero as $\lambda \rightarrow-\infty$.

Proof of (b). Let $\psi \in \dot{H}^{2}\left(\Sigma_{\lambda}\right) \cap \dot{W}_{0}^{1, \frac{2 n}{n-2}}\left(\Sigma_{\lambda}\right)$ fulfill the assumptions of Lemma 9. Then the odd extension of $\psi$,

$$
\varphi(x)= \begin{cases}\psi(x) & \text { if } x \in \Sigma_{\lambda} \\ -\psi\left(x^{\lambda}\right) & \text { if } x \in \Sigma_{\lambda}^{c}\end{cases}
$$

belongs to $\dot{H}^{2}\left(\mathbb{R}^{n}\right)$ and is harmonic near $S$. Therefore, Lemma 8 and a straightforward change of variables yield

$$
\begin{aligned}
& \int_{\Sigma_{\lambda}} \Delta w \Delta \psi=\int_{\mathbb{R}^{n}} \Delta v \Delta \varphi=\int_{\mathbb{R}^{n}} k^{\frac{n+4}{2}} g\left(k^{-\frac{n-4}{2}} v\right) \varphi \\
= & \int_{\Sigma_{\lambda}}\left(k^{\frac{n+4}{2}} g\left(k^{-\frac{n-4}{2}} v\right)-k_{\lambda}^{\frac{n+4}{2}} g\left(v_{\lambda} k_{\lambda}^{-\frac{n-4}{2}}\right)\right) \psi \\
= & \int_{\Sigma_{\lambda}} V w \psi+\int_{\Sigma_{\lambda}}\left(k^{\frac{n+4}{2}} g\left(k^{-\frac{n-4}{2}} v\right)-k_{\lambda}^{\frac{n+4}{2}} g\left(v k_{\lambda}^{-\frac{n-4}{2}}\right)\right) \psi \\
\geqslant & \int_{\Sigma_{\lambda}} V w \psi \geqslant-\int_{\Sigma_{\lambda}} V w_{-} \psi .
\end{aligned}
$$

In the first inequality, we used $k \geqslant k_{\lambda}$ on $\Sigma_{\lambda}$ together with the fact that the function $s \mapsto s^{\frac{n+4}{2}} g\left(v s^{-\frac{n-4}{2}}\right)$ is non-decreasing on $(0, \infty)$ for every fixed $v>0$, which follows from the inequality $g^{\prime}(t) \leqslant \frac{n+4}{n-4} \frac{g(t)}{t}$ in assumption (5). In the second inequality, we used $V \geqslant 0$ and $\psi \geqslant 0$. 
We can now derive the crucial technical ingredient for the moving planes method from inequality (18). The use of such bounds in the context of the method of moving planes goes back at least to [6].

Lemma 10. Let $v, g$ and $k$ fulfill the assumptions of Theorem 5 and let $w^{(\lambda)}=v-v_{\lambda}$ on $\Sigma_{\lambda}$. Let $V^{(\lambda)}$ be defined by (17). Then there is $\epsilon_{0}>0$, depending only on $n$, such that if $\left|\left\{w^{(\lambda)}<0\right\}\right|>0$, then $\int_{\left\{w^{(\lambda)}<0\right\}}\left(V^{(\lambda)}\right)^{\frac{n}{4}} \geqslant \epsilon_{0}$.

Proof. We abbreviate $w=w^{(\lambda)}$ and $V=V^{(\lambda)}$. We claim that the assertion follows if we can prove the following two inequalities,

$$
\begin{aligned}
\left(\int_{\Sigma_{\lambda}} w_{-}^{\frac{2 n}{n-4}}\right)^{\frac{n-4}{2 n}} & \lesssim\left(\int_{\Sigma_{\lambda}}(-\Delta w)_{-}^{2}\right)^{\frac{1}{2}} \\
\left(\int_{\Sigma_{\lambda}}(-\Delta w)_{-}^{2}\right)^{\frac{1}{2}} & \lesssim\left(\int_{\{w<0\}} V^{\frac{n}{4}}\right)^{\frac{4}{n}}\left(\int_{\Sigma_{\lambda}} w_{-}^{\frac{2 n}{n-4}}\right)^{\frac{n-4}{2 n}}
\end{aligned}
$$

with implied constants depending only on $n$. Indeed, (201) and (21) together yield the bound

$$
\left(\int_{\Sigma_{\lambda}} w_{-}^{\frac{2 n}{n-4}}\right)^{\frac{n-4}{2 n}} \lesssim\left(\int_{\{w<0\}} V^{\frac{n}{4}}\right)^{\frac{4}{n}}\left(\int_{\Sigma_{\lambda}} w_{-}^{\frac{2 n}{n-4}}\right)^{\frac{n-4}{2 n}} .
$$

If $w_{-} \not \equiv 0$, we may divide by $\left(\int_{\Sigma_{\lambda}} w_{-}^{\frac{2 n}{n-4}}\right)^{\frac{n-4}{2 n}} \neq 0$ to deduce the bound

$$
1 \lesssim\left(\int_{\{w<0\}} V^{\frac{n}{4}}\right)^{\frac{4}{n}}
$$

which concludes the proof of Lemma 10, Thus, it remains to prove inequalities (20) and (21).

Before proving these inequalities, let us note that the second factor on the right side of (21) is finite since $w \geqslant-v_{\lambda}$ and $v_{\lambda} \in L^{\frac{2 n}{n-4}}\left(\Sigma_{\lambda}\right)$ by our assumption on $v$. Moreover, by Lemma 9 the first factor on the right side of (21) is finite and therefore it is part of the assertion of this inequality that $(-\Delta w)_{-} \in L^{2}\left(\Sigma_{\lambda}\right)$.

For any $f \in L^{2}\left(\Sigma_{\lambda}\right)$, we can define

$$
\psi(x):=c_{n} \int_{\Sigma_{\lambda}}\left(\frac{1}{|x-y|^{n-2}}-\frac{1}{\left|x-y^{\lambda}\right|^{n-2}}\right) f(y), \quad x \in \Sigma_{\lambda},
$$

with $c_{n}=\left((n-2)\left|\mathbb{S}^{n-1}\right|\right)^{-1}$. Then we have $\psi \in \dot{H}^{2}\left(\Sigma_{\lambda}\right) \cap \dot{W}_{0}^{1, \frac{2 n}{n-2}}\left(\Sigma_{\lambda}\right)$ and $-\Delta \psi=f$ on $\Sigma_{\lambda}$.

Notice that $f \leqslant 0$ implies that $\psi \leqslant 0$ on $\Sigma_{\lambda}$. Moreover, formula (22) and the HardyLittlewood-Sobolev inequality [5, Theorem 4.3] imply the bound

$$
\|\psi\|_{L^{r}\left(\Sigma_{\lambda}\right)} \lesssim\|f\|_{L^{s}\left(\Sigma_{\lambda}\right)}
$$

for every pair of exponents $1<s<r<\infty$ related by $\frac{n-2}{n}+\frac{1}{s}=1+\frac{1}{r}$. 
We now give the proofs of the two inequalities (20) and (21).

To prove (20), for every $k \in \mathbb{N}$ and $x \in \Sigma_{\lambda} \backslash S$, set

$$
f_{k}(x)=-w_{-}^{\frac{n+4}{n-4}}(x) 1_{\left\{w_{-} \leqslant k\right\}}(x) 1_{\left\{\operatorname{dist}(x, S) \geqslant k^{-1},|x| \leqslant k\right\}}(x) .
$$

Thus, $f_{k}$ is bounded and has compact support and, in particular, $f_{k} \in L^{2}\left(\Sigma_{\lambda}\right)$. We consider $\psi_{k}$ associated to $f_{k}$ by (22). Notice that $-\Delta \psi_{k}=f_{k} \equiv 0$ in a neighborhood of $S$.

We have, by dominated (or monotone) convergence,

$$
\int_{\Sigma_{\lambda}} w_{-}^{\frac{2 n}{n-4}}=\lim _{k \rightarrow \infty} \int_{\Sigma_{\lambda}} w f_{k}=\lim _{k \rightarrow \infty} \int_{\Sigma_{\lambda}} w\left(-\Delta \psi_{k}\right)
$$

Let us introduce a family of cutoff functions $\eta_{\epsilon}$ as in (14), but now vanishing both near 0 and $a$. Since $\psi_{k}$ and $w$ vanish on $\partial \Sigma_{\lambda}$, integration by parts gives

$$
\int_{\Sigma_{\lambda}} w\left(-\Delta\left(\psi_{k} \eta_{\epsilon}\right)\right)=\int_{\Sigma_{\lambda}}(-\Delta w)\left(\psi_{k} \eta_{\epsilon}\right) \leqslant-\int_{\Sigma_{\lambda}}(-\Delta w)_{-}\left(\psi_{k} \eta_{\epsilon}\right) .
$$

Here the inequality holds simply because $\psi_{k} \leqslant 0$. As $\epsilon \rightarrow 0$, the right hand side of (24) tends to $-\int_{\Sigma_{\lambda}}(-\Delta w)_{-} \psi_{k}$, by monotone convergence. Moreover, since $\psi_{k}$ is harmonic in a neighborhood of $S$, we can argue as in the proof of Lemma 8 that the left hand side of (24) tends to $\int_{\Sigma_{\lambda}} w\left(-\Delta \psi_{k}\right)$ as $\epsilon \rightarrow 0$.

We therefore have that

$$
\int_{\Sigma_{\lambda}} w\left(-\Delta \psi_{k}\right) \leqslant-\int_{\Sigma_{\lambda}}(-\Delta w)_{-} \psi_{k} \leqslant\left\|(-\Delta w)_{-}\right\|_{2}\left\|\psi_{k}\right\|_{2} \lesssim\left\|(-\Delta w)_{-}\right\|_{2}\left\|f_{k}\right\|_{\frac{2 n}{n+4}} .
$$

The second inequality here is Hölder's inequality and the third one uses (23) with $r=2, s=\frac{2 n}{n+4}$. Letting $k \rightarrow \infty$, we find that

$$
\int_{\Sigma_{\lambda}} w_{-}^{\frac{2 n}{n-4}} \lesssim\left\|(-\Delta w)_{-}\right\|_{2}\left\|w_{-}^{\frac{n+4}{n-4}}\right\|_{\frac{2 n}{n+4}}=\left\|(-\Delta w)_{-}\right\|_{2}\left\|w_{-}\right\|_{\frac{2 n}{n-4}}^{\frac{n+4}{n-4}},
$$

which implies inequality (20) since $\left\|w_{-}\right\|_{\frac{2 n}{n-4}}<\infty$ as we have noted before.

To prove (21), for every $k \in \mathbb{N}$ and $x \in \Sigma_{\lambda} \backslash S$, set

$$
f_{k}(x)=-(-\Delta w)_{-}(x) 1_{\left\{(-\Delta w)_{-} \leqslant k\right\}} 1_{\left\{\operatorname{dist}(x, S) \geqslant k^{-1},|x| \leqslant k\right\}} .
$$

Thus, $f_{k}$ is bounded and has compact support and, in particular, $f_{k} \in L^{2}\left(\Sigma_{\lambda}\right)$. We consider the associated $\psi_{k}$ as above and notice that $-\Delta \psi_{k}=f_{k} \equiv 0$ in a neighborhood of $S$.

We have, by monotone convergence,

$$
\int_{\Sigma_{\lambda}}(-\Delta w)_{-}^{2}=-\lim _{k \rightarrow \infty} \int_{\Sigma_{\lambda}} \Delta w f_{k}=\lim _{k \rightarrow \infty} \int_{\Sigma_{\lambda}} \Delta w \Delta \psi_{k}
$$


Since $-\psi_{k} \geqslant 0$ fulfills all the assumptions of Lemma 9, by (18) we obtain

$$
\begin{aligned}
\int_{\Sigma_{\lambda}} \Delta w \Delta \psi_{k} \leqslant-\int_{\Sigma_{\lambda}} V w_{-} \psi_{k} & \leqslant\left\|V 1_{\{w<0\}}\right\| \frac{n}{4}\left\|w_{-}\right\|_{\frac{2 n}{n-4}}\left\|\psi_{k}\right\|_{\frac{2 n}{n-4}} \\
& \lesssim\left\|V 1_{\{w<0\}}\right\| \frac{n}{4}\left\|w_{-}\right\|_{\frac{2 n}{n-4}}\left\|f_{k}\right\|_{2},
\end{aligned}
$$

where we used Hölder's inequality followed by (23) again. Since

$$
\int_{\Sigma_{\lambda}} \Delta w \Delta \psi_{k}=\left\|f_{k}\right\|_{2}^{2}<\infty
$$

we deduce that

$$
\left\|f_{k}\right\|_{2} \lesssim\left\|V 1_{\{w<0\}}\right\|_{\frac{n}{4}}\left\|w_{-}\right\|_{\frac{2 n}{n-4}}
$$

for all $k \in \mathbb{N}$. Passing to the limit $k \rightarrow \infty$, we obtain inequality (21) by monotone convergence.

We are finally in a position to prove Theorem 5 .

Proof of Theorem 5. By rotation invariance we may assume that $a_{1}=0$ and $H=$ $\left\{x_{1}=0\right\}$. For $\lambda<0$, we consider the function $w^{(\lambda)}=v-v_{\lambda}$, defined on $\Sigma_{\lambda}$, and $V^{(\lambda)}$ given by (17). By Lemma 9, we have $\int_{\left\{w^{(\lambda)}<0\right\}}\left(V^{(\lambda)}\right)^{\frac{n}{4}}<\epsilon_{0}$ for all $\lambda$ sufficiently negative. Therefore Lemma 10 implies that $w^{(\lambda)} \geqslant 0$ on $\Sigma_{\lambda}$ for all $\lambda$ sufficiently negative.

Therefore

$$
\lambda_{0}=\sup \left\{\lambda<0: w^{(\mu)} \geqslant 0 \text { for all } \mu<\lambda\right\},
$$

is well-defined.

We claim that either $w_{\lambda_{0}}>0$ a.e. on $\Sigma_{\lambda_{0}}$ or $w_{\lambda_{0}} \equiv 0$. Indeed, by continuity, we still have $w^{\left(\lambda_{0}\right)} \geqslant 0$ a.e. in $\Sigma_{\lambda_{0}}$. Moreover, inequality (21) implies that $-\Delta w^{(\lambda)} \geqslant 0$ in $\Sigma_{\lambda}$ for $\lambda<\lambda_{0}$ and, therefore, by continuity $-\Delta w^{\left(\lambda_{0}\right)} \geqslant 0$ in $\Sigma_{\lambda_{0}}$. (For the continuity argument, we pass to the limit in the inequality $\int_{\Sigma_{\lambda}} w^{(\lambda)}(-\Delta) \varphi \geqslant 0$ for $0 \leqslant \varphi \in$ $C_{0}^{\infty}\left(\Sigma_{\lambda}\right)$.) The claim now follows by the strong maximum principle in $\Sigma_{\lambda_{0}}$.

After these preliminaries we now show that if $\lambda_{0}<0$, then $w^{\left(\lambda_{0}\right)} \equiv 0$. Later we will see that $w^{\left(\lambda_{0}\right)} \equiv 0$ is impossible and therefore we will conclude that $\lambda_{0}=0$. We argue by contradiction and assume that $\lambda_{0}<0$ and that $w^{\left(\lambda_{0}\right)} \not \equiv 0$. Then by the above argument $w^{\left(\lambda_{0}\right)}>0$ a.e. on $\Sigma_{\lambda_{0}}$. This strict inequality implies that the quantity

$$
I(\lambda)=\int_{\left\{x \in \Sigma_{\lambda}: w^{(\lambda)}(x)<0\right\}}\left(v_{\lambda}^{\frac{2 n}{n-4}}+k_{\lambda}^{n}\right)
$$

tends to zero as $\lambda \searrow \lambda_{0}$. Indeed, any sequence of $\lambda$ 's tending to $\lambda_{0}$ has a subsequence along which $w^{(\lambda)}(x) \rightarrow w^{\left(\lambda_{0}\right)}(x)$ pointwise for a.e. $x \in \Sigma_{\lambda_{0}}$. Since $w^{\left(\lambda_{0}\right)}>0$, we have 
that $1_{\left\{w^{(\lambda)}<0\right\}}(x) \rightarrow 0$ pointwise for a.e. $x \in \Sigma_{\lambda_{0}}$, which is the same as $1_{\left\{v(x)>v\left(x^{\lambda}\right)\right\}} \rightarrow 0$ pointwise almost everywhere in $\left\{x_{1}<\lambda_{0}\right\}$. Therefore,

$$
I(\lambda)=\int_{\left\{x_{1}<\lambda\right\}} 1_{\left\{v(x)>v\left(x^{\lambda}\right)\right\}}(x)\left(v^{\frac{2 n}{n-4}}(x)+k^{n}(x)\right) \rightarrow 0
$$

by dominated convergence, in view of the integrability assumptions on $v$ and $k$ and the assumption $\lambda_{0}<0$.

On the other hand, as in the proof of Lemma 9, we have $\int_{\left\{w^{(\lambda)}<0\right\}}\left(V^{(\lambda)}\right)^{\frac{n}{4}} \lesssim I(\lambda)$ for all $\lambda<0$. This, together with $I(\lambda) \rightarrow 0$ as $\lambda \searrow \lambda_{0}$, implies by Lemma 10 that there is a $\delta>0$ such that $w^{(\lambda)} \geqslant 0$ for all $\lambda \in\left(\lambda_{0}, \lambda_{0}+\delta\right)$. This is a contradiction to the definition of $\lambda_{0}$, and therefore we conclude that $w^{\left(\lambda_{0}\right)} \equiv 0$ if $\lambda_{0}<0$.

We now show that $w^{\left(\lambda_{0}\right)} \equiv 0$ for $\lambda_{0}<0$ is impossible under the assumptions of the theorem. Indeed, if $v \equiv v_{\lambda_{0}}$ and $\lambda_{0}<0$, then, by the integrability assumption on $v$,

$$
\int_{\mathbb{R}^{n}} v^{\frac{2 n}{n-4}}=\int_{x_{1}<\lambda_{0}} v^{\frac{2 n}{n-4}}+\int_{x_{1}>\lambda_{0}} v^{\frac{2 n}{n-4}}=2 \int_{x_{1}<\lambda_{0}} v^{\frac{2 n}{n-4}}<\infty .
$$

This is impossible under the assumption $v \notin L^{\frac{2 n}{n-4}}\left(\mathbb{R}^{n}\right)$. Also, if $v \equiv v_{\lambda_{0}}$, then by (8) , $k^{\frac{n+4}{2}} g\left(k^{-\frac{n-4}{2}} v\right) \equiv k_{\lambda}^{\frac{n+4}{2}} g\left(k_{\lambda}^{-\frac{n-4}{2}} v\right)$. Thus,

$0=k^{\frac{n+4}{2}} g\left(k^{-\frac{n-4}{2}} v\right)-k_{\lambda}^{\frac{n+4}{2}} g\left(k_{\lambda}^{-\frac{n-4}{2}} v\right)=\frac{n-4}{2} \int_{k_{\lambda}}^{k}\left(\frac{n+4}{n-4} \frac{g\left(s^{-\frac{n-4}{2}} v\right)}{s^{-\frac{n-4}{2}} v}-g^{\prime}\left(s^{-\frac{n-4}{2}} v\right)\right) v s^{3}$

and when the integrand is positive for almost every $s$, then we deduce that $k \equiv k_{\lambda}$. To summarize, under the assumptions of the theorem it is impossible that $w^{\left(\lambda_{0}\right)} \equiv 0$ for $\lambda_{0}<0$. Thus, we conclude that $\lambda_{0}=0$.

The fact that $\lambda_{0}=0$ implies that

$$
v\left(x_{1}, x_{2}, \ldots, x_{n}\right) \geqslant v\left(-x_{1}, x_{2}, \ldots, x_{n}\right) \text { for all } x_{1}>0, x_{2}, \ldots, x_{n} \in \mathbb{R}
$$

and that $v$ is strictly increasing in $x_{1}$ on $\left\{x_{1}<0\right\}$. (The strictness follows from the fact that $w^{(\lambda)}>0$ for $\lambda<0$.)

Considering $\tilde{v}\left(x_{1}, x_{2}, \ldots, x_{n}\right)=v\left(-x_{1}, x_{2}, \ldots, x_{n}\right)$, which solves the same equation as $v$ due to the symmetry of $k$, and repeating all of the previous arguments, we derive the complementary inequality

$$
v\left(x_{1}, x_{2}, \ldots, x_{n}\right) \leqslant v\left(-x_{1}, x_{2}, \ldots, x_{n}\right) \text { for all } x_{1}>0, x_{2}, \ldots, x_{n} \in \mathbb{R}
$$

and the fact that $v$ is strictly decreasing in $x_{1}$ on $\left\{x_{1}>0\right\}$.

Hence $v$ is strictly symmetric-decreasing with respect to $\left\{x_{1}=0\right\}$, as claimed. 


\section{ODE ANALYSIS}

In this section, we prove Theorem 3 as well as Remark 2 .

Recall from the introduction that by the radial symmetry of any solution $u$ on $\mathbb{R}^{n} \backslash\{0\}$ of (11) proved in Theorem 1, we can define a function $v$ on $\mathbb{R}$ by setting $u(x)=$ $|x|^{-\frac{n-4}{2}} v(\ln |x|)$. Via this Emden-Fowler change of variables, equation (11) is equivalent to

$$
v^{(4)}-A v^{\prime \prime}+B v=g(v) \quad \text { in } \mathbb{R}
$$

for certain constants $A$ and $B$, depending on $n$. The only property of these constants that we will be using is that

$$
A^{2}>4 B>0 \quad \text { and } \quad A>0 .
$$

In [3] we have classified all entire solutions (i.e., solutions defined on all of $\mathbb{R}$ ) of equation (25) in the case $g(v)=v^{\frac{n+4}{n-4}}$. In the present section we extend this classification to all $g$ satisfying the assumptions (5). In a complementary way to the proof of Theorem 5, in this section we actually make no use of the upper bound $g(t) / t \leqslant(n+4) /(n-4) g^{\prime}(t)$ from (5) .

Our exposition here will focus on the main steps of the argument, providing details only where there is a significant difference to [3].

3.1. Proof of Theorem 3. We will assume throughout this section that $g$ satisfies the assumptions (5). Let us introduce

$$
G(v)=\int_{0}^{v} g(t) \mathrm{d} t-B \frac{v^{2}}{2} .
$$

The crucial observation is that for the proofs in [3] only some qualitative properties of $G$ are needed, as described in the next lemma.

Lemma 11. One has $G(0)=G^{\prime}(0)=0$. Moreover, there is an $a_{0}>0$ such that $G$ is strictly decreasing on $\left(0, a_{0}\right)$ and strictly increasing towards $\infty$ on $\left(a_{0}, \infty\right)$.

Proof. The first part follows directly from (27) and the fact that $\lim _{t \rightarrow 0} g(t)=0$.

For the second part, we write $G^{\prime}=g(v)-B v=v\left(\frac{g(v)}{v}-B\right)$. The assumptions (5) imply that $\frac{g(v)}{v}-B$ is negative for all sufficiently small $v$ and that $v \mapsto \frac{g(v)}{v}$ is strictly increasing. Thus, there is an $a_{0}>0$ such that $G^{\prime}$ is strictly negative on $\left(0, a_{0}\right)$ and strictly positive on $\left(a_{0}, \infty\right)$. From the assumption $g(t) \geqslant c t^{q}$ in (5) we obtain $G(v) \geqslant \frac{c}{q+1} v^{q+1}-\frac{B}{2} v^{2}-C \rightarrow \infty$ as $v \rightarrow \infty$.

The following lemma concerns the asymptotic behavior of entire solutions. Its most notable consequence is that any solution to (25) which tends to $\infty$ must blow up in finite time. 
Lemma 12. Let $v \in C^{4}(\mathbb{R})$ be a positive solution of (25) and suppose that $a:=$ $\lim _{t \rightarrow \infty} v(t) \in[0, \infty]$ exists. Then either $a=0$ or $a=a_{0}$.

If one uses Lemma 11 and the inequality $g(t) \geqslant c t^{q}$ from (5), the proof is analogous to that of [4, Lemma 2.1], which treats the special case $g(v)=v^{\frac{n+4}{n-4}}$. We omit the details.

The following comparison lemma is a key technical ingredient in our argument. It is a variant of [7, Lemma 1], see also [1, Theorem 2.1]. The novelty here is that it is stated and proved for positive solutions, instead of bounded solutions. This difference allows to significantly shorten the proof in [3], because boundedness of entire solutions, which was one of the main steps in [3], need no longer be shown a priori.

For the statement of the lemma we recall the structural assumption (26), which implies that the polynomial $\xi^{2}-A \xi+B$ has two distinct positive roots. We denote these by $\lambda>\mu>0$.

Lemma 13. Let $v, w \in C^{4}(\mathbb{R})$ be positive solutions to the equation (25) with

$$
\begin{aligned}
v(0) & \geqslant w(0), \\
v^{\prime}(0) & \geqslant w^{\prime}(0), \\
v^{\prime \prime}(0)-\mu v(0) & \geqslant w^{\prime \prime}(0)-\mu w(0), \\
v^{\prime \prime \prime}(0)-\mu v^{\prime}(0) & \geqslant w^{\prime \prime \prime}(0)-\mu w^{\prime}(0) .
\end{aligned}
$$

Then $v \equiv w$.

Because of its importance for us, we include a complete proof of this lemma. It follows closely that of [7, Lemma 1], but uses in addition Lemma [12.

Proof. Let $v$ and $w$ satisfy the assumptions of the lemma and suppose, by contradiction, that $v \not \equiv w$. Then by uniqueness of ODE solutions and by our hypotheses on the initial conditions, there is $k \in\{0,1,2,3\}$ such that $v^{(k)}(0)>w^{(k)}(0)$ and $v^{(l)}(0)=w^{(l)}(0)$ for all $0 \leqslant l<k$. Therefore, in any case,

$$
v>w \text { on }(0, \sigma)
$$

for some sufficiently small $\sigma>0$.

We define the auxiliary functions

$$
\phi(t):=v^{\prime \prime}(t)-\mu v(t) \quad \text { and } \quad \psi(t):=w^{\prime \prime}(t)-\mu w(t) .
$$

Then by the hypotheses, we have

$$
(\phi-\psi)(0) \geqslant 0 \text { and }(\phi-\psi)^{\prime}(0) \geqslant 0 .
$$

From equation (25) and by the definition of $\lambda$ and $\mu$, we have

$$
(\phi-\psi)^{\prime \prime}(t)-\lambda(\phi-\psi)(t)=g(v(t))-g(w(t))
$$


for all $t \in \mathbb{R}$. Because of (28) and the fact that $g$ is strictly increasing on $(0, \infty)$, this implies that

$$
(\phi-\psi)^{\prime \prime}(t)-\mu(\phi-\psi)(t)>0 \quad \text { for all } t \in(0, \sigma) .
$$

The inequalities (29) and (30) easily imply that $(\phi-\psi)(t) \geqslant 0$ for $t \in(0, \sigma)$, or equivalently, that

$$
(v-w)^{\prime \prime}(t) \geqslant \lambda(v-w)(t) \quad \text { for all } t \in(0, \sigma) .
$$

Since $(v-w)^{\prime}(0) \geqslant 0$ by the hypotheses of the lemma, we see from (31) and (28) that $(v-w)^{\prime}(t)>0$ for all $t \in(0, \sigma)$. Hence $v-w$ is strictly increasing on $(0, \sigma)$ and, since $\sigma>0$ was arbitrary with the property (28), we infer that $v-w$ remains strictly positive for all times.

Repeating the above arguments for the interval $(0, \infty)$ instead of $(0, \sigma)$, we see from (31) and (28) that $(v-w)^{\prime}$ is positive and strictly increasing on $(0, \infty)$. Thus $\lim _{t \rightarrow \infty}(v-$ $w)(t)=\infty$. Since $w$ is positive, this implies, in particular, that $\lim _{t \rightarrow \infty} v(t)=\infty$. This contradicts Lemma 12, and we have therefore proved that $v \equiv w$.

From Lemma 13 we can deduce a remarkable rigidity property, namely that positive entire solutions to (25) are determined by only two (instead of four!) initial values. A simple consequence of this is that positive solutions of (25) are symmetric with respect to local extrema.

Corollary 14. (i) Let $v, w \in C^{4}(\mathbb{R})$ be positive solutions of (25) with $v(0)=w(0)$ and $v^{\prime}(0)=w^{\prime}(0)$. Then $v \equiv w$.

(ii) Suppose that $v \in C^{4}(\mathbb{R})$ is a positive solution of (25) with $v^{\prime}\left(t_{0}\right)=0$ for some $t_{0} \in \mathbb{R}$. Then $v$ is symmetric with respect to $t_{0}$, i.e. $v\left(t_{0}+t\right)=v\left(t_{0}-t\right)$ for all $t \in \mathbb{R}$.

We point out once more that we only assume positivity of $v$ in Corollary 14, whereas in [3, Corollary 5] we assumed boundedness of $v$.

Proof of Corollary 14. To prove $(i)$, we observe that up to exchanging $v$ and $w$, we may assume $v^{\prime \prime}(0) \geqslant w^{\prime \prime}(0)$. Furthermore, up to replacing $v(t)$ and $w(t)$ by $v(-t)$ and $w(-t)$ (which still solve (25) ), we may assume $v^{\prime \prime \prime}(0) \geqslant w^{\prime \prime \prime}(0)$. Then all assumptions of Lemma 13 are satisfied and we conclude $v \equiv w$.

To prove $(i i)$, we simply apply $(i)$ to $v$ and $w(t)=v\left(t_{0}-t\right)$, which also solves (25).

We now prove a variant of Lemma 13 where one of the functions is constant.

Lemma 15. Let $v \in C^{4}(\mathbb{R})$ be a positive solution of (25) and assume that either

$$
v(0) \geqslant a_{0}, \quad v^{\prime}(0)=0, \quad v^{\prime \prime}(0) \geqslant 0, \quad v^{\prime \prime \prime}(0)=0
$$


or

$$
v(0) \leqslant a_{0}, \quad v^{\prime}(0)=0, \quad v^{\prime \prime}(0) \leqslant 0, \quad v^{\prime \prime \prime}(0)=0
$$

Then $v \equiv a_{0}$.

Proof. Proof under the first set of assumptions. Suppose, by contradiction, that $v \neq \equiv$ $a_{0}$. Then by uniqueness of ODE solutions, either $v(0)>a_{0}$ or $v^{\prime \prime}(0)>0$. Moreover, from the equation, we have

$$
v^{(4)}(t)=A v^{\prime \prime}(t)+(g(v(t))-B v(t)) .
$$

Observing that $g(v)-B v>0$ for $v \in\left(a_{0}, \infty\right)$, we deduce that in both cases $\left(v(0)>a_{0}\right.$ or $\left.v^{\prime \prime}(0)>0\right)$ we have $v^{(4)}>0$ on $(0, \sigma)$ for some sufficiently small $\sigma>0$.

Together with the initial conditions, this implies that $v^{(k)}$ is strictly increasing on $(0, \sigma)$ for $k=0,1,2,3$. Since $\sigma>0$ was arbitrary with the property that the right side of (32) is positive, we infer, in particular, that $v^{\prime}$ is positive and strictly increasing on $(0, \infty)$. Thus $\lim _{t \rightarrow \infty} v(t)=\infty$. This contradicts Lemma 12, and we have therefore proved that $v \equiv a_{0}$.

Proof under the second set of assumptions. Suppose, by contradiction, that $v \neq \equiv a_{0}$. Then by uniqueness of ODE solutions, either $v(0)<a_{0}$ or $v^{\prime \prime}(0)<0$.

Observing that $g(v)-B v<0$ for $v \in\left(0, a_{0}\right)$, we deduce from (32) that in both cases $\left(v(0)<a_{0}\right.$ or $\left.v^{\prime \prime}(0)<0\right)$ we have $v^{(4)}<0$ on $(0, \sigma)$ for some sufficiently small $\sigma>0$.

Together with the initial conditions, this implies that $v^{(k)}$ is strictly decreasing on $(0, \sigma)$ for $k=0,1,2,3$. Since $\sigma>0$ was arbitrary with the property that the right side of (32) is negative, we infer, in particular, that $v^{\prime}$ is negative and strictly decreasing as long as $v>0$. Therefore, we must have $v\left(t_{0}\right)=0$ with $v^{\prime}\left(t_{0}\right)<0$ for some finite $t_{0}<\infty$. This contradicts the positivity of $v$ and we have therefore proved that $v \equiv a_{0}$.

At last, we give the main ideas for the proofs of Theorems 3 and 4 using the ingredients introduced so far.

Proof sketch of Theorem [3. By arguments detailed in [3, Proof of Proposition 3], we can deduce from Lemmas 12 and 13 that every positive solution $v \in C^{4}(\mathbb{R})$ is either constant equal to $a_{0}$, homoclinic to zero or periodic with unique local maximum and minimum per period.

We can now prove $(i)$. The existence and uniqueness of $a_{0}$ with the claimed properties is contained in Lemma 11. Next, let $v \in C^{4}(\mathbb{R})$ be a positive solution with $\min _{\mathbb{R}} v \geqslant$ $a_{0}$. Suppose without loss that $v(0)=\min _{\mathbb{R}} v$. Then $v^{\prime}(0)=0, v^{\prime \prime}(0) \geqslant 0$ and, by Corollary 14, $v$ is symmetric, so $v^{\prime \prime \prime}(0)=0$. Thus, Lemma 15 implies $v \equiv a_{0}$. This completes the proof of $(i)$. 
The existence part of assertion $(i i)$ can be proved via the shooting method, identically to [3]. We invite the reader to check that by the properties of $G$ stated in Lemma 13, all of the argument carries over to the more general case considered here. For the uniqueness part of assertion $(i i)$ we use the fact mentioned above that any solution $v$ with $\inf _{\mathbb{R}} v=a>0$ is either constant or periodic (since it cannot be homoclinic to zero). In particular, $\inf _{\mathbb{R}} v$ is attained. Therefore the uniqueness follows from the first part of Corollary 14. The stated periodicity and monotonicity properties follow from the second part of Corollary 14 .

Finally, we prove $(i i i)$. We obtain the existence of a homoclinic solution simply as a limit of periodic solutions. Indeed, if we denote by $v_{a}$ the periodic solutions obtained in $(i i)$ with the normalization $v_{a}(0)=\max v_{a}$ and by $L_{a}$ their period length, then $v_{a}$ is symmetric-decreasing on $\left(-\frac{L_{a}}{2}, \frac{L_{a}}{2}\right)$. Using $L_{a} \rightarrow \infty$ as $a \rightarrow 0$, it is not difficult to prove that $v_{a}$ converges to a non-trivial limit function which must be the homoclinic solution. See Subsection 3.2 for details.

Next, we prove the uniqueness claim in $($ iii $)$. We first note that if $v$ is a positive solution with $\inf _{\mathbb{R}} v=0$, then $v$ is homoclinic to zero. This follows from the fact mentioned above, since if $v$ periodic or constant, it cannot be positive and have $\inf _{\mathbb{R}} v=0$. Now let $v$ and $w$ be two positive solutions in $C^{4}(\mathbb{R})$ with the property that $v^{\prime}(0)=w^{\prime}(0)=0$ and $\lim _{|t| \rightarrow \infty} v(t)=\lim _{|t| \rightarrow \infty} w(t)=0$. We argue by contradiction and assume $v \not \equiv$ $w$. Then, by the first part of Corollary [14, we may assume that $v(0)>w(0)$. By comparison arguments detailed in [3, Lemma 9], this enforces that $v(t)>w(t)$ for all $t \in \mathbb{R}$.

We can now derive the desired contradiction. For every $R>0$, we have, using integration by parts and the fact that $v$ and $w$ satisfy (25),

$$
\begin{aligned}
0 & =\int_{-R}^{R} w\left(v^{(4)}-A v^{\prime \prime}+B v-g(v)\right) \\
& =b(R)+\int_{-R}^{R} v\left(w^{(4)}-A w^{\prime \prime}+B w-g(w)\right)+\int_{-R}^{R} w v\left(\frac{g(w)}{w}-\frac{g(v)}{v}\right) \\
& =b(R)+\int_{-R}^{R} w v\left(\frac{g(w)}{w}-\frac{g(v)}{v}\right) .
\end{aligned}
$$

Here, $b(R)$ contains all the boundary terms coming from the integrations by part. As in [7, Lemma 4] one shows that $b(R) \rightarrow 0$ as $R \rightarrow \infty$. But since the function $t \mapsto \frac{g(t)}{t}$ is strictly increasing on $(0, \infty)$ and since $v>w$, we find that $\int_{-R}^{R} w v\left(\frac{g(w)}{w}-\frac{g(v)}{v}\right)$ is a negative and strictly decreasing function of $R$. Thus we obtain a contradiction by choosing $R$ large enough.

Finally, the claimed decay behavior can be proved by relatively standard comparison arguments, again relying on the factorization structure of equation (25). See Subsection 3.3 for details. This concludes the proof of Theorem 3 . 
Proof of Remark 国. The inequality $\frac{\partial u}{\partial|x|}<0$ is equivalent to the bound $v^{\prime}<\frac{n-4}{2} v$. Similarly as in the proof of Lemma 13 we introduce $\mu$ and $\lambda$ and

$$
\phi(t):=v^{\prime \prime}(t)-\mu v(t),
$$

which satisfies

$$
\phi^{\prime \prime}-\lambda \phi=g(v) .
$$

(We emphasize that here $\mu=(n-4)^{2} / 4$, which is potentially different from the use of $\mu$ in Theorem 33) Since $g(v)>0$, it follows from the maximum principle that $\phi<0$ on $\mathbb{R}$. Indeed, by Theorem 3 we know that $v$ is either constant, periodic or homoclinic to zero. In the first two cases the maximum principle can be clearly applied. In the third case it can be applied since $\lim _{|t| \rightarrow \infty} \phi(t)=0$, as we already argued in the proof of Theorem 3 .

The function $w:=v^{\prime} / v$ satisfies

$$
w^{\prime}=-w^{2}+\mu+\frac{\phi}{v} .
$$

Since $\phi<0$ and $v>0$, we have

$$
w^{\prime}<-w^{2}+\mu
$$

In particular, $w^{\prime}(t)<0$ whenever $|w(t)| \geqslant \sqrt{\mu}$. This implies that the set $\{w \geqslant \sqrt{\mu}\}$ is either empty, or equal to $\mathbb{R}$ or of the form $(-\infty, T]$ for some $T \in \mathbb{R}$. We will rule out the last two possibilities and conclude that $w<\sqrt{\mu}$, as claimed.

We make again use of the classification result from Theorem 3, When $v$ is constant or periodic, there is a two-sided sequence $\left(t_{n}\right)_{n \in \mathbb{Z}}$ with $t_{n} \rightarrow \pm \infty$ as $n \rightarrow \pm \infty$ such that $v^{\prime}\left(t_{n}\right)=0$ for all $n$. This implies $w\left(t_{n}\right)=0$ and therefore the set in question can neither be of the form $\mathbb{R}$ nor of the form $(-\infty, T]$ for $T \in \mathbb{R}$.

Now assume that $v$ is homoclinic to zero. Then there is a $t_{0} \in \mathbb{R}$ such that $v^{\prime}\left(t_{0}\right)=0$ and therefore the set cannot be of the form $\mathbb{R}$. Now suppose that there is a $T \in \mathbb{R}$ such that $w(t) \geqslant \sqrt{\mu}$ for all $t \leqslant T$. By (35)), $w^{\prime}<0$ on $(-\infty, T]$. Therefore there is an $\epsilon>0$ such that $w(t) \geqslant \sqrt{\mu+\epsilon}$ for all $t \leqslant T-1=: T_{1}$. Thus, $\mu \leqslant(\mu /(\mu+\epsilon)) w(t)^{2}$ and so (35) implies $w^{\prime}<-(\epsilon /(\mu+\epsilon)) w^{2}$ on $\left(-\infty, T_{1}\right)$ and therefore by integration,

$$
\frac{1}{w\left(T_{1}\right)}-\frac{1}{w(t)}>\frac{\epsilon}{\mu+\epsilon}\left(T_{1}-t\right) \quad \text { for all } t<T_{1} .
$$

Since $w(t)>0$, this is a contradiction for $t$ sufficiently negative. This completes the proof.

3.2. Existence of a homoclinic solution. In this subsection we provide the details in the existence part of (iii) in Theorem 3. As already explained, we shall construct a homoclinic solution to (25) as a limit of periodic solutions $v_{a}$ with $a \searrow 0$. 
The family $\left(v_{a}\right)_{a \in\left(0, a_{0}\right)}$ of periodic solutions and their associated minimal period lengths $\left(L_{a}\right)_{a \in\left(0, a_{0}\right)}$ were introduced before the statement of Theorem 4 . We recall that $v_{a}(0)=$ $\max _{\mathbb{R}} v_{a}$, that $v_{a}\left( \pm \frac{L_{a}}{2}\right)=\min _{\mathbb{R}} v_{a}=a$ and that $v_{a}$ is strictly symmetric-decreasing on $\left(-\frac{L_{a}}{2}, \frac{L_{a}}{2}\right)$.

The following lemma is fundamental for our construction.

Lemma 16. Let $a_{n} \searrow 0$ and consider $v_{n}=v_{a_{n}}$ with associated minimal period length $L_{n}=L_{a_{n}}$. Then there is $v_{\infty} \in C^{4}(\mathbb{R})$ such that, up to extracting a subsequence, we have $v_{0} \rightarrow v_{\infty}$ in $C^{4}(K)$ for every compact $K \subset \mathbb{R}$. Moreover, $v_{\infty}(0) \geqslant a_{0}$ and, in particular, $v_{\infty} \neq \equiv 0$.

Proof. Fix $R>0$ and consider the compact interval $[-R, R]$. We shall prove that

$$
\sup _{n \in \mathbb{N}} \sup _{t \in[-R, R]}\left(\left|v_{n}(t)\right|+\left|v_{n}^{\prime \prime}(t)\right|\right)<\infty .
$$

Indeed, if (36) holds, then by equation (25),$v_{n}^{(4)}=A v_{n}^{\prime \prime}-B v_{n}+g\left(v_{n}\right)$ is also bounded on $[-R, R]$ and so are $v_{n}^{\prime}(t)=\int_{0}^{t} v_{n}^{\prime \prime}(s) \mathrm{d} s$ and $v_{n}^{\prime \prime \prime}(t)=\int_{0}^{t} v_{n}^{(4)}(s) \mathrm{d} s$. By Arzelà-Ascoli, up to a subsequence, we thus have $v_{n} \rightarrow v_{\infty}$ in $C^{3}([-R, R])$ and, by using the equation again, in $C^{4}([-R, R])$. Since $R>0$ was arbitrary, we conclude by a diagonal argument.

To prove (36), we consider the energy

$$
\mathcal{E}_{v}(t)=-v^{\prime}(t) v^{\prime \prime \prime}(t)+\frac{v^{\prime \prime}(t)^{2}}{2}+A \frac{v^{\prime}(t)^{2}}{2}+G(v(t)) .
$$

By differentiating and using (25) we see that $\mathcal{E}_{v}(t)$ is constant with respect to $t$. Moreover, by [7, Corollary 6], we have

$$
\frac{v_{n}^{\prime \prime}(t)^{2}}{2}+G\left(v_{n}(t)\right) \leqslant \mathcal{E}_{v_{n}}
$$

for all $n \in \mathbb{N}$. Since $G(v) \rightarrow \infty$ as $v \rightarrow \infty$, boundedness of both $v_{n}^{\prime \prime}(t)$ and $v_{n}(t)$ will follow if we can prove that $\mathcal{E}_{v_{n}}$ is bounded uniformly in $n$.

To do so, we claim that $v_{n}^{\prime \prime}\left(\frac{L_{a_{n}}}{2}\right) \leqslant b / A$, where $b:=\max _{v \in[0, \infty)}(B v-g(v))$. (We recall from the proof of Lemma 11 that $0<b<\infty$.) Indeed, if this bound on $v_{n}^{\prime \prime}\left(\frac{L_{a}}{2}\right)$ was not true, the initial conditions and the equation

$$
v_{n}^{(4)}=A v_{n}^{\prime \prime}-B v_{n}+g\left(v_{n}\right)
$$

would imply that $v_{n}^{(4)}$ is positive, and $v_{n}^{\prime \prime}$ is increasing for all times. However, this is impossible because $v_{n}$ is periodic.

Thus, since $v_{n}\left(\frac{L_{a_{n}}}{2}\right)=a_{n} \in\left(0, a_{0}\right)$ and therefore $G\left(v_{n}\left(\frac{L_{a_{n}}}{2}\right)\right)<0$, we have

$$
\mathcal{E}_{v_{n}}=\mathcal{E}_{v_{n}}\left(\frac{L_{a_{n}}}{2}\right)=\frac{v_{n}^{\prime \prime}\left(\frac{L_{a_{n}}}{2}\right)^{2}}{2}+G\left(v_{n}\left(\frac{L_{a_{n}}}{2}\right)\right) \leqslant \frac{b^{2}}{2 A^{2}}
$$

for all $n \in \mathbb{N}$. This finishes the proof of (36). 
Lastly, we prove that $v_{\infty}(0) \geqslant a_{0}$. We first note that the inequality $v_{a}(0)>a_{0}$ follows from Lemma 15, similarly as in the proof of $(i)$ in Theorem 3. Letting $a \rightarrow 0$, we find $v_{\infty}(0) \geqslant a_{0}$.

The second observation that we need is that the period length diverges as the minimum value approaches 0 .

Lemma 17. As $a \rightarrow 0, L_{a} \rightarrow \infty$.

Proof. Suppose that there is a sequence $a_{n} \backslash 0$ and $L_{\infty}<\infty$ such that $L_{n}:=L_{a_{n}} \rightarrow$ $L_{\infty}$. Then by Lemma 16, up to a subsequence, there is a nonnegative $v_{\infty} \in C^{4}(\mathbb{R})$ which solves (25) such that $v_{n}:=v_{a_{n}} \rightarrow v_{\infty}$ in $C^{4}\left(\left[-L_{\infty}, L_{\infty}\right]\right)$. Moreover, we have $v_{\infty}\left(\frac{L_{\infty}}{2}\right)=\lim _{n \rightarrow \infty} v_{n}\left(\frac{L_{n}}{2}\right)=\lim _{n \rightarrow \infty} a_{n}=0$ and $v_{\infty}^{\prime}\left(\frac{L_{\infty}}{2}\right)=\lim _{n \rightarrow \infty} v_{n}^{\prime}\left(\frac{L_{n}}{2}\right)=0$. From Lemma 13, we deduce that $v_{\infty} \equiv 0$, in contradiction to Lemma 16.

We can now prove the desired existence result.

Lemma 18. There is a positive solution $v_{0} \in C^{4}(\mathbb{R})$ of $(25)$ with $\lim _{|t| \rightarrow \infty} v_{0}(t)=0$.

Proof. By Lemma 16, there is a nonnegative solution $v_{0} \in C^{4}(\mathbb{R})$ of (25) such that $v_{n} \rightarrow v_{0}$ in $C^{4}(K)$ for every compact $K \subset \mathbb{R}$. Since $v_{n}$ is symmetric-decreasing on $\left[-\frac{L_{n}}{2}, \frac{L_{n}}{2}\right]$, and since $L_{n} \rightarrow \infty$ by Lemma [17, $v_{0}$ is symmetric-decreasing on all of $\mathbb{R}$ and therefore $\lim _{t \rightarrow \infty} v_{0}(t)$ exists. By Lemma 12, this limit equals either 0 or $a_{0}$ and it remains to exclude the second case.

Thus, suppose that $\lim _{t \rightarrow \infty} v_{0}(t)=a_{0}$. We can derive a contradiction using the energy $\mathcal{E}_{v}$ introduced in the proof of Lemma 16. Using the fact that all derivatives of $v_{0}$ vanish at $\infty$ by [7, Lemma 4] (note that $v_{0}$ is monotone as required for this lemma), we have

$$
\mathcal{E}_{v_{0}}=\lim _{t \rightarrow \infty} \mathcal{E}_{v_{0}}(t)=G\left(a_{0}\right)<0 .
$$

On the other hand, we have for each $n$,

$$
\mathcal{E}_{v_{n}}=\mathcal{E}_{v_{n}}\left(\frac{L_{a_{n}}}{2}\right)=\frac{v_{n}^{\prime \prime}\left(\frac{L_{a_{n}}}{2}\right)^{2}}{2}+G\left(v_{n}\left(\frac{L_{a_{n}}}{2}\right)\right)=\frac{v_{n}^{\prime \prime}\left(\frac{L_{a_{n}}}{2}\right)^{2}}{2}+G\left(a_{n}\right) \geqslant G\left(a_{n}\right) .
$$

Thus, since $v_{n} \rightarrow v_{0}$ in $C^{4}(K)$ for any compact $K$ implies $\mathcal{E}_{v_{n}} \rightarrow \mathcal{E}_{v_{0}}$ as $n \rightarrow \infty$ and since $G\left(a_{n}\right) \rightarrow G(0)=0$ as $n \rightarrow \infty$, we have

$$
\mathcal{E}_{v_{\infty}}=\lim _{n \rightarrow \infty} \mathcal{E}_{v_{n}} \geqslant \lim _{n \rightarrow \infty} G\left(a_{n}\right)=0,
$$

contradicting (37). This contradiction shows that $\lim _{t \rightarrow \infty} v_{0}(t)=0$. By the symmetry of $v_{0}$, we also obtain $\lim _{t \rightarrow-\infty} v_{0}(t)=0$ and the proof is complete. 
3.3. Decay behavior of the homoclinic solution. We prove the following decay behavior of the homoclinic solution of (25). We recall that we assume $0 \leqslant \beta=$ $\lim _{s \rightarrow 0} \frac{g(s)}{s}<B$ and set $\mu=\frac{1}{2}\left(A-\sqrt{A^{2}-4(B-\beta)}\right)$.

Lemma 19. Let $v \in C^{4}(\mathbb{R})$ be a positive solution of (25) with $\lim _{|t| \rightarrow \infty} v(t)=0$. Then for every $\epsilon>0$ there is a $C_{\epsilon}<\infty$ such that $v(t) \leqslant C_{\epsilon} e^{-(\sqrt{\mu}-\epsilon)|t|}$ for all $t \in \mathbb{R}$.

The proof of this bound relies on a comparison argument using the following fourthorder variant of the maximum principle.

Lemma 20. Suppose that $w \in C^{4}(\mathbb{R})$ satisfies the inequality

$$
\left(\frac{\mathrm{d}^{2}}{\mathrm{~d} t^{2}}-\lambda\right)\left(\frac{\mathrm{d}^{2}}{\mathrm{~d} t^{2}}-\mu\right) w(t) \geqslant 0 \quad \text { on }(T, \infty)
$$

for some $\lambda, \mu>0$ and $T \in \mathbb{R}$ and that $w(T)=w^{\prime \prime}(T)=\lim _{t \rightarrow \infty} w(t)=\lim _{t \rightarrow \infty} w^{\prime \prime}(t)=$ 0 . Then $w \geqslant 0$ on $(T, \infty)$.

Proof. The inequality for $w$ factorizes into the system

$$
\left\{\begin{array}{l}
w^{\prime \prime}-\lambda w=u \\
u^{\prime \prime}-\mu u \geqslant 0
\end{array}\right.
$$

By assumption, we have $u(T)=0$ and $\lim _{t \rightarrow \infty} u(t)=0$. By the maximum principle applied to the inequality $u^{\prime \prime}-\mu u \geqslant 0$, we thus deduce that

$$
w^{\prime \prime}-\lambda w=u \leqslant 0 \text {. }
$$

By assumption, we have $w(T)=0$ and $\lim _{t \rightarrow \infty} w(t)=0$. Applying the maximum principle a second time, we deduce $w \geqslant 0$, as desired.

Proof of Lemma 19. We first observe that the limit behavior of $g$ and $g^{\prime}$ at 0 given by (5), together with the mean value theorem easily imply that $\lim _{v \rightarrow 0} \frac{g(v)}{v}$ exists and is equal to $\beta=\lim _{v \rightarrow 0} g^{\prime}(v)$. Therefore, we may write $g(v)=\beta v+h(v)$ with $\lim _{v \rightarrow 0} \frac{h(v)}{v}=0$ and think of equation (25) as

$$
v^{(4)}-A v^{\prime \prime}+(B-\beta) v=h(v)
$$

Fix some $\epsilon>0$ such that $B-\beta-\epsilon>0$ and $T_{\epsilon}$ such that $\frac{h(v(t))}{v(t)} \leqslant \epsilon$ for all $t>T_{\epsilon}$. Since $v>0$, equation (38) implies

$$
v^{(4)}-A v^{\prime \prime}+(B-\beta-\epsilon) v \leqslant 0 \quad \text { on }\left(T_{\epsilon}, \infty\right) .
$$

Since by our choice of $\epsilon$, the condition $A^{2}>4(B-\beta-\epsilon)>0$ is still satisfied, the expression on the left hand side factorizes as

$$
\left(\frac{\mathrm{d}^{2}}{\mathrm{~d} t^{2}}-\lambda_{\epsilon}\right)\left(\frac{\mathrm{d}^{2}}{\mathrm{~d} t^{2}}-\mu_{\epsilon}\right) v(t) \leqslant 0 \quad \text { on }\left(T_{\epsilon}, \infty\right)
$$

with some $\lambda_{\epsilon}>\mu_{\epsilon}>0$. 
We compare $v$ with a solution $f_{\epsilon}$ of

$$
\left(\frac{\mathrm{d}^{2}}{\mathrm{~d} t^{2}}-\lambda_{\epsilon}\right)\left(\frac{\mathrm{d}^{2}}{\mathrm{~d} t^{2}}-\mu_{\epsilon}\right) f_{\epsilon}=0 .
$$

and which tends to zero as $t \rightarrow \infty$. The general solution of this problem is given by

$$
f_{\epsilon}(t)=a_{\epsilon} e^{-\sqrt{\lambda_{\epsilon}} t}+b_{\epsilon} e^{-\sqrt{\mu_{\epsilon}} t}, \quad a_{\epsilon}, b_{\epsilon} \in \mathbb{R} .
$$

We may fix $a_{\epsilon}$ and $b_{\epsilon}$ such that

$$
f_{\epsilon}\left(T_{\epsilon}\right)=v\left(T_{\epsilon}\right) \quad \text { and } \quad f_{\epsilon}^{\prime \prime}\left(T_{\epsilon}\right)=v^{\prime \prime}\left(T_{\epsilon}\right) .
$$

(This is always possible as a consequence of $\lambda_{\epsilon} \neq \mu_{\epsilon}$ ). Since moreover, $\lim _{t \rightarrow \infty} v^{\prime \prime}(t)=0$ by [7, Lemma 4], the function $w:=f_{\epsilon}-v$ fulfills all assumptions from Lemma 20. We therefore deduce that $w \geqslant 0$, i.e., $v \leqslant f_{\epsilon}=O\left(e^{-\sqrt{\mu_{\epsilon}} t}\right)$ as $t \rightarrow \infty$. Analogously, one obtains $v=O\left(e^{\sqrt{\mu_{\epsilon}} t}\right)$ as $t \rightarrow-\infty$. Since $\mu_{\epsilon} \rightarrow \mu$ as $\epsilon \rightarrow 0$, we obtain the claimed bound.

Lemma 21. Let $v \in C^{4}(\mathbb{R})$ be a positive solution of $(25)$ with $\lim _{|t| \rightarrow \infty} v(t)=0$ and assume that $g$ satisfies the additional assumption (17). Then $\lim _{|t| \rightarrow \infty} e^{\sqrt{\mu}|t|} v(t)$ exists, is positive and finite.

Proof. Equation (38) reads, in factorized form,

$$
\left(\frac{\mathrm{d}^{2}}{\mathrm{~d} t^{2}}-\lambda\right)\left(\frac{\mathrm{d}^{2}}{\mathrm{~d} t^{2}}-\mu\right) v=h(v)
$$

with $\lambda>\mu>0$ and given by

$$
\lambda=\frac{A+\sqrt{A^{2}-4(B-\beta)}}{2} \quad \text { and } \quad \mu=\frac{A-\sqrt{A^{2}-4(B-\beta)}}{2} .
$$

The Green's function associated to equation (39) is

$$
G(t, s)=\frac{1}{\lambda-\mu}\left(\frac{e^{-\sqrt{\mu}|t-s|}}{2 \sqrt{\mu}}-\frac{e^{-\sqrt{\lambda}|t-s|}}{2 \sqrt{\lambda}}\right) .
$$

According to (7) and Lemma 19 we have

$$
|h(v(t))| \leqslant C_{\epsilon} e^{-r(\sqrt{\mu}-\epsilon)|t|}
$$

As a consequence, we can solve equation (39) for $v$ using $G$ and obtain

$$
v(t)=\int_{\mathbb{R}} G(t, s) h(v(s)) .
$$

Using this formula and (41) with $\epsilon>0$ chosen so small that $r(\sqrt{\mu}-\epsilon)>\sqrt{\mu}$ it is easy to deduce that

$$
\lim _{t \rightarrow \infty} e^{\sqrt{\mu} t} v(t)=\frac{1}{\lambda-\mu} \frac{1}{2 \sqrt{\mu}} \int_{\mathbb{R}} e^{\sqrt{\mu} s} h(v(s)),
$$

where the right side is finite. Moreover, if $h \geqslant 0$, then the limit is positive. (Note that (5) implies that $h(v) / v<h^{\prime}(v)$ for all $v>0$ and therefore $h$ is non-zero.) 


\section{REFERENCES}

[1] B. Buffoni, A. R. Champneys, and J. F. Toland. Bifurcation and coalescence of a plethora of homoclinic orbits for a Hamiltonian system. J. Dynam. Differential Equations, 8(2):221-279, 1996.

[2] Luis A. Caffarelli, Basilis Gidas, and Joel Spruck. Asymptotic symmetry and local behavior of semilinear elliptic equations with critical Sobolev growth. Comm. Pure Appl. Math., 42(3):271297, 1989.

[3] Rupert L. Frank and Tobias König. Classification of positive singular solutions to a nonlinear biharmonic equation with critical exponent. Anal. PDE, 12(4):1101-1113, 2019.

[4] Z. Guo, X. Huang, L. Wang, and J. Wei. On Delaunay solutions of a biharmonic elliptic equation with critical exponent. ArXiv e-prints, August 2017.

[5] Elliott H. Lieb and Michael Loss. Analysis, volume 14 of Graduate Studies in Mathematics. American Mathematical Society, Providence, RI, second edition, 2001.

[6] Susanna Terracini. Symmetry properties of positive solutions to some elliptic equations with nonlinear boundary conditions. Differential Integral Equations, 8(8):1911-1922, 1995.

[7] Jan Bouwe van den Berg. The phase-plane picture for a class of fourth-order conservative differential equations. J. Differential Equations, 161(1):110-153, 2000.

[8] X. Xu. Uniqueness theorem for the entire positive solutions of biharmonic equations in $\mathbf{R}^{n}$. Proc. Roy. Soc. Edinburgh Sect. A, 130(3):651-670, 2000.

[9] Hui Yang. Asymptotic behavior of positive solutions to a nonlinear biharmonic equation near isolated singularities. arXiv e-prints, page arXiv:1812.06555, Dec 2018.

(Rupert L. Frank) Mathematisches Institut, Ludwig-Maximilans Universität MÜnchen, Theresienstr. 39, 80333 München, Germany, and Mathematics 253-37, Caltech, PasaDENA, CA 91125, USA

E-mail address: r.frank@lmu.de, rlfrank@caltech.edu

(Tobias König) Mathematisches Institut, Ludwig-Maximilans Universität MÜnchen, ThereSienstr. 39, 80333 MÜnChen, Germany

E-mail address: tkoenig@math.lmu.de 